Pacific

Journal of

Mathematics

HAMILTONIAN EVOLUTIONS OF TWISTED POLYGONS IN PARABOLIC MANIFOLDS:

THE LAGRANGIAN GRASSMANNIAN

GLORIA MARÍ BeFFA 


\title{
HAMILTONIAN EVOLUTIONS OF TWISTED POLYGONS IN PARABOLIC MANIFOLDS: THE LAGRANGIAN GRASSMANNIAN
}

\author{
GLORIA MARÍ BEFFA
}

\begin{abstract}
We show that the moduli space of twisted polygons in $G / P$, where $G$ is semisimple and $\boldsymbol{P}$ parabolic, and where $\mathfrak{g}$ has two coordinated gradations has a natural Poisson bracket that is directly linked to $G$-invariant evolutions of polygons. This structure is obtained by reducing the quotient twisted bracket on $G^{N}$ (as defined by M. Semenov-Tian-Shansky) to the moduli space $G^{N} / P^{N}$. We prove that any Hamiltonian evolution with respect to this bracket is induced on $G^{N} / P^{N}$ by an invariant evolution of polygons. We describe in detail the Lagrangian Grassmannian case $(G=$ $\operatorname{Sp}(2 n))$ and we describe a submanifold of Lagrangian subspaces where the reduced bracket becomes a decoupled system of Volterra Hamiltonian structures. We also describe a very simple evolution of polygons whose invariants evolve following a decoupled system of Volterra equations.
\end{abstract}

\section{Introduction}

The difference geometry of lattices, although a relatively young subject, has been known to be related to completely integrable systems almost from its conception. Indeed, parallel to the well-known fact that the sine-Gordon equation describes surfaces with constant negative Gauss curvature, the work of Bobenko and others on difference geometry of lattices (see for instance [Bobenko and Suris 2008]) consistently relates certain types of 2-lattices to completely integrable lattice systems. While in the continuous case the sine-Gordon equation appears as the CodazziMainardi equation of the surface in appropriately chosen coordinates, in the lattice case they are described as the compatibility condition of special types of lattices, with the different properties of lattices playing the role of specially chosen coordinates.

More recently, a flurry of work on the pentagram map, its generalizations and related subjects (see, for example, [Ovsienko et al. 2010; Marí Beffa 2013; Khesin and Soloviev 2013], although the bibliography on this subject is quite vast) has

Supported in part by NSF grant DMS \#0804541 and the Simons Foundation.

MSC2010: 39AXX.

Keywords: discrete Hamiltonian systems, discrete Lagrangian Grassmannian, Hamiltonian evolutions of polygons in parabolic manifolds, discrete Poisson reduction. 
clearly pointed at a relation between dynamics of polygons, rather than 2-lattices, and completely integrable systems. Indeed the pentagram map, a map defined on projective planar polygons (both twisted and closed), was proven to be completely integrable and a discretization of the Boussinesq equation when written in terms of the discrete projective invariants of the polygons [Ovsienko et al. 2010; 2013; Soloviev 2013].

A plethora of work in the continuous case takes us in this same direction when one works with curves rather than surfaces. Most, if not all, well-known completely integrable PDEs have been realized as systems induced on differential invariants by a flow of curves in some homogeneous manifold. For example, the KdV equation is induced on the Schwarzian derivative of a flow in $\mathbb{R P}^{1}$ solution of the so-called Schwarzian-KdV equation (this is a classical result that one can check by hand). Similarly Adler-Gel'fand-Dikii flows are induced on projective differential invariants of flows in $\mathbb{R P}^{n}$ by some known evolutions (see [Marí Beffa 1999]). Likewise the literature shows realizations of mKdV [Terng and Thorbergsson 2001], NLS [Terng and Uhlenbeck 2006] Sawada-Kotera [Chou and Qu 2002], modified Sawada-Kotera [Chou and Qu 2003], vector sine-Gordon [Wang 2002] and most other well-known systems as flows of curves in several different manifolds. This list is by no means exhaustive and many equations are realized as a curve flow in more than one geometry; see, for example, [Calini et al. 2009; Chou and Qu 2002; 2003].

Inspired by the recent developments in discrete maps, we studied in [Mansfield et al. 2013] the relation between evolutions of twisted polygons in homogeneous manifolds and completely integrable lattice systems on the geometric invariants of the flow. In particular we found an evolution of projective planar polygons that when written in terms of projective curvatures becomes a modified Volterra lattice. We also found realizations of the Toda lattice as evolution of polygons in the centro-affine plane; an integrable discretization of the Toda lattice induced by a centro-affine map; and a realization of a Volterra-type equation as evolution of polygons on the homogeneous 2-sphere. In [Marí Beffa and Wang 2013] we proved that one can obtain a Hamiltonian structure on the moduli space of twisted polygons in $\mathbb{R P}^{n}$ through the reduction of a twisted Poisson bracket on lattices defined by Semenov-Tian-Shansky [1985], and that any Hamiltonian with respect to the reduced bracket was induced on invariants by an evolution of polygons in $\mathbb{R} \mathbb{P}^{n}$, with the gradient of the Hamiltonian defining the evolution in a direct and simple fashion. The reduced bracket was a Hamiltonian structure for an integrable discretization of $W_{n}$-algebras, and this discretization was induced on projective invariants by a rather simple polygon evolution. We also found a second structure for the system via reduction of the right bracket, a structure that was not originally Poisson. 
This paper can be viewed as a second part to [Marí Beffa and Wang 2013]. Here we consider the case of polygons in $G / P$, with $G$ semisimple, $P$ parabolic, and $\mathfrak{g}$ a $|1|$-graded algebra (and Lie algebra with parabolic gradation $\mathfrak{g}=\mathfrak{g}_{1} \oplus \mathfrak{g}_{0} \oplus \mathfrak{g}_{-1}$, $\mathfrak{p}=\mathfrak{g}_{1} \oplus \mathfrak{g}_{0}$ ). These include many of the well-known nonaffine geometries (conformal $n$-sphere, $\mathbb{R P}^{n}$, Grassmannians, Lagrangian Grassmannians, pure spinors and other flag manifolds). We assume that the parabolic gradation is coordinated with a second gradation of the form $\mathfrak{g}_{+} \oplus \mathfrak{h} \oplus \mathfrak{g}_{-}$, with $\mathfrak{h}$ commutative. We prove that the twisted Poisson bracket of Semenov-Tian-Shansky defined and associated to this second gradation can also be reduced to the moduli space of polygons in $G / P$ resulting on a natural Poisson structure on the space of polygon invariants. We also prove that there are simple ways to connect the reduced Hamiltonian structure to evolutions of polygons with evolutions inducing Hamiltonian systems on the invariants of the flow. In particular, we prove that any Hamiltonian evolution is induced on invariants by an evolution of polygons in $G / P$. This result is valid also in more general settings, and we discuss this fact in our last section.

We study in detail the example of the Lagrangian Grassmannian, that is, polygons of Lagrangian subspaces in $\mathbb{R}^{2 n}, M=\mathrm{Sp}(2 n) / P$, with $P$ a parabolic subgroup. We find an appropriate discrete moving frame along twisted polygons, and we define the Schwarzian difference of Lagrangian planes (a discrete analogue of the Schwarzian derivative defined in [Ovsienko 1993].) The frame provides us with a complete description of the invariants and produces a generating set that includes the eigenvalues of the Schwarzian difference. We then apply our general theorem to find a Hamiltonian structure on the space of invariants associated to our moving frame. We show that the reduced Poisson bracket can be reduced once more to the space of polygons for which the nonschwarzian invariants are equal to the identity, and we show that this reduction decouples into a system of $n$ second Hamiltonian structures for the Volterra chain [Khanizadeh et al. 2013]. Using this information we define evolutions of Lagrangian planes inducing the Volterra chain on the eigenvalues of the Schwarzian difference of the flow. The continuous analogue of this study can be found in [Marí Beffa 2007].

Section 2 includes background definitions and results that will be used in the paper, both in the subject of discrete moving frames and on Poisson Lie groups and Semenov-Tian-Shansky's bracket. Section 3 proves the existence of the Poisson bracket on the moduli space (as represented by the discrete invariants) and its relation to the Sklyanin bracket (Theorem 3.4). In Section 4 we describe in detail the direct relation between polygon evolutions and reduced Hamiltonians; in particular we prove that any Hamiltonian is induced on invariants by a polygon evolution and we give the direct connection between both (Theorem 4.2). We study the Lagrangian Grassmannian in Section 5 while Section 6 summarizes the paper and discusses generalizations to other homogeneous manifolds and some open 
problems. Recall that in the projective case [Marí Beffa and Wang 2013] the Poisson structures obtained in the planar case were not preserved by the pentagram map (the bihamiltonian nature of the map is still an open problem, as far as we know). This was also pointed out by Marshall [2010].

\section{Background and definitions}

As a starting point we will give a brief description of discrete moving frames and their associated invariants. The description is taken from [Mansfield et al. 2013] and can also be found in [Marí Beffa and Wang 2013], but we include it here for completeness.

Discrete moving frames. Let $G$ be a Lie group and let $\mathfrak{g}$ be its Lie algebra (it can be real or complex). Let $M$ be a manifold and let $G \times M \rightarrow M$ be the action of the group $G$ on $M$.

Definition 2.1 (twisted $N$-gon). A twisted $N$-gon in $M$ is a map $\phi: \mathbb{Z} \rightarrow M$ such that $\phi(p+N)=g \cdot \phi(p)$ for some fixed $g \in G$ and for all $p \in \mathbb{Z}$. (The dot notation represents the action of $G$ on $M$.) The element $g \in G$ is called the monodromy of the polygon. We will denote a twisted $N$-gon by its image $x=\left(x_{s}\right)$, where $x_{s}=\phi(s)$.

The main reason to work with twisted polygons is our desire to work with periodic invariants (in order to have a finite number of them). One could restrict further to closed polygons, but since the solution of a periodic discrete equation is, in general, twisted, restricting to closed polygons creates additional technical problems we would like to avoid here. We will denote by $P_{N}$ the space of twisted $N$-gons in $M$. Clearly $P_{N} \cong M^{N}$, and since $G$ acts on $M$, it also acts on $P_{N}$ with the diagonal action $g \cdot\left(x_{s}\right)=\left(g \cdot x_{s}\right)$.

Definition 2.2 (discrete moving frame). Let $G^{N}$ denote the Cartesian product of $N$ copies of the group $G$. Elements of $G^{N}$ will be denoted by $\left(g_{s}\right)$. Allow $G$ to act on the right of $G^{N}$ using the inverse diagonal action $g \cdot\left(g_{s}\right)=\left(g_{s} g^{-1}\right)$ (respectively left, using the diagonal action $\left.g \cdot\left(g_{s}\right)=\left(g g_{s}\right)\right)$. We say a map

$$
\rho: P_{N} \rightarrow G^{N}
$$

is a right (respectively left) discrete moving frame if $\rho$ is equivariant with respect to the diagonal action of $G$ on $P_{N}$ and the right inverse (respectively left) diagonal action of $G$ on $G^{N}$. Whenever $\rho(x) \in G^{N}$, we will denote by $\rho_{s}$ its $s$-th component; that is $\rho=\left(\rho_{s}\right)$, where $\rho_{s}(x) \in G$ for all $s, x=\left(x_{s}\right)$. Clearly, if $\rho=\left(\rho_{s}\right)$ is a right moving frame, then $\rho^{-1}=\left(\rho_{s}^{-1}\right)$ is a left moving frame, and vice versa. Thus, a moving frame associates an element of the group to each vertex of the polygon in 
an equivariant fashion. In our examples the moving frame will be invariant under the shift $\tau x_{s}=x_{s+1}$, but this need not be the case in general.

Proposition 2.3 [Mansfield et al. 2013]. Let $\mathscr{b}$ be a collection $\mathscr{C}_{1}, \ldots, \mathscr{C}_{N}$ of local cross-sections to the orbit of $G$ through $x_{1}, \ldots, x_{N}$. Let $\rho=\left(\rho_{s}\right) \in G$ be uniquely determined by the condition

$$
\rho_{s} \cdot\left(x_{r}\right) \in \mathscr{C}_{s}
$$

for any $s$. Then $\rho=\left(\rho_{S}\left(\left(x_{r}\right)\right)\right) \in G^{N}$ is a right moving frame along the $N$-gon $\left(x_{r}\right)$.

Discrete moving frames carry the invariant information of the polygon, as we see next.

Definition 2.4 (discrete invariant). Let $I: P_{N} \rightarrow \mathbb{R}$ be a function defined on $N$-gons. We say that $I$ is a scalar discrete invariant if

$$
I\left(\left(g \cdot x_{s}\right)\right)=I\left(\left(x_{s}\right)\right)
$$

for any $g \in G$ and any $x=\left(x_{s}\right) \in P_{N}$.

We will naturally refer to vector invariants when considering vectors whose components are scalar invariants. Although not necessary, for simplicity of notation we will assume from now on that $G \subset \operatorname{GL}(n, \mathbb{R})$. Nevertheless, results are also true for some exceptional Lie algebras, as we will see later.

Definition 2.5 (Maurer-Cartan matrix). Let $\rho$ be a right (respectively left) discrete moving frame evaluated along a twisted $N$-gon. The element of the group

$$
K_{s}=\rho_{s+1} \rho_{s}^{-1} \quad\left(\text { respectively } \rho_{s}^{-1} \rho_{s+1}\right)
$$

is called the right (respectively left) $s$-Maurer-Cartan matrix for $\rho$. We will call the equation $\rho_{s+1}=K_{s} \rho_{s}$ the right $s$-Serret-Frenet equation (respectively $\rho_{s+1}=\rho_{s} K_{s}$ is the left one). The element $K=\left(K_{s}\right) \in G^{N}$ is called the right (respectively left) Maurer-Cartan matrix for $\rho$.

One can directly check that if $K=\left(K_{s}\right)$ is a Maurer-Cartan matrix for the right frame $\rho$, then $\left(K_{s}^{-1}\right)$ is a left one for the left frame $\rho^{-1}=\left(\rho_{s}^{-1}\right)$, and vice versa. The entries of a Maurer-Cartan matrix are functional generators of all discrete invariants of polygons, as it was shown in [Mansfield et al. 2013]. This fact is an immediate consequence of the following recursion formulas: Let's denote by $\rho_{r} \cdot x_{s}=I_{s}^{r}$ the so-called basic invariants. One can check directly from the definitions that if $K$ is a right Maurer-Cartan matrix, then

$$
K_{r} \cdot I_{s}^{r}=I_{s}^{r+1}
$$


for any $r, s$. The basic invariants with $r$ fixed generate other invariants since from (2), if $I$ is an invariant,

$$
I\left(\left(\rho_{r} \cdot x_{s}\right)\right)=I\left(\left(x_{s}\right)\right)=I\left(I_{r}^{s}\right) .
$$

From this and (3), one concludes that the entries of $K_{s}$ are generators also (see [Mansfield et al. 2013]).

Assume next that $M=G / H$, with $G$ acting on $M$ via left multiplication on representatives of the class. Let us denote by $o \in M$ the class of $H$.

The following theorem, which can be found in [Mansfield et al. 2013], describes how to write a general invariant polygon evolution in terms of moving frames. Denote by $\Phi_{g}: G / H \rightarrow G / H$ the map defined by the action of $g \in G$ on $G / H$, that is, $\Phi_{g}(x)=g \cdot x$.

Theorem 2.6. Let $\rho$ be a right moving frame, and for simplicity assume that $\rho_{s} \cdot x_{s}=o$ for all s. Any $G$-invariant evolution can be written as

$$
\left(x_{s}\right)_{t}=d \Phi_{\rho_{s}^{-1}}(o)\left(\boldsymbol{v}_{s}\right),
$$

where $\boldsymbol{v}_{S}(x) \in T_{x_{S}} M$ is an invariant vector.

Notice that if, in general, $\rho_{s} \cdot x_{s}=y_{s} \neq o$, one can easily change $\rho_{s}$ to $\hat{\rho}_{s}=g_{s} \rho_{s}$, where $g_{s} \cdot y_{s}=o$ (the action is transitive). The frame $\hat{\rho}_{s}$ will also be a right moving frame and thus one can always find a frame with the condition given by the theorem. This fact will greatly simplify both notations and calculations.

If a family of polygons $x(t)$ is evolving according to (4), there is a simple process to describe the evolution induced on the Maurer-Cartan matrices and hence on a generating set of invariants. It is described in the following theorem, which can also be found in [Mansfield et al. 2013], slightly modified.

Before our next theorem, let us settle some notation and choices. Assume

$$
\mathfrak{g}=\mathfrak{m} \oplus \mathfrak{h}
$$

where $\mathfrak{m}$ is a linear complement to $\mathfrak{h}$. Consider $\varsigma: G / H \rightarrow G$ to be a section of $G / H$ such that $\varsigma(o)=e \in G$ and $\mathfrak{m}$ is the tangent to the image of $\varsigma$. Let $\rho$ be a right moving frame coordinated with $\varsigma$. That is, assume $\rho_{s} \cdot x_{s}=o$ so that $\rho_{s}=\rho_{s}^{H} \varsigma\left(x_{s}\right)^{-1}$, for some $\rho_{s}^{H} \in H$.

Let $K_{s}$ be a right Maurer-Cartan matrix and define $N_{s}=\left(\rho_{s}\right)_{t} \rho_{s}^{-1} \in \mathfrak{g}$ to describe the time evolution of the frame.

Theorem 2.7. Assume $x(t)$ is a flow of polygons solution of (4). Then

$$
\left(K_{s}\right)_{t}=N_{s+1} K_{s}-K_{s} N_{s}
$$

and, if $N_{s}=N_{s}^{\mathfrak{h}}+N_{s}^{\mathfrak{m}}$ splits according to (5), then

$$
N_{s}^{\mathfrak{m}}=-d \varsigma(o) v_{s} .
$$


In most examples Equation (6) and condition (7) completely determine $N$ and the evolution of $K$, even if we do not know the moving frame explicitly. This will be clear later in our Lagrangian example.

Finally, in [Marí Beffa and Wang 2013], we proved the following theorem, which is true for any homogeneous manifold. Assume we have a nondegenerate twisted polygon $x=\left(x_{s}\right)$ in a manifold $M=G / H$ with associated right moving frame $\rho$ such that $\rho_{s} \cdot x_{s}=o$ for all $s$. By nondegenerate we mean a polygon for which a moving frame can be constructed, but we can also think of generic cases. (It was shown in [Boutin 2002] that generically a moving frame always exists for $N$ large enough.) Let us assume that the subgroup $H^{N}$ acts naturally on $G^{N}$ via the gauge transformation

$$
\left(g_{s}\right) \rightarrow\left(h_{s+1} g_{s} h_{s}^{-1}\right)
$$

(assuming $h_{s+N}=h_{s}$ for all $s$ ).

Theorem 2.8. In a neighborhood of a nondegenerate polygon, the right MaurerCartan matrices $K$ associated to right moving frames $\rho$ describe a section of the quotient $G^{N} / H^{N}$. That is, let $x \in G^{N} / H^{N}$ be a nondegenerate twisted polygon, U with $x \in \mathcal{U}$ an open set of $G^{N} / H^{N}$ containing nondegenerate twisted polygons, and let $\mathscr{H}$ be the set of all the Maurer-Cartan matrices in $G^{N}$ associated to right moving frames for elements in $U$ and determined by a fixed transverse section as in Proposition 2.3. Then the map

$$
\mathscr{K} \rightarrow G^{N} / H^{N}, \quad\left(K_{S}\right) \rightarrow\left[\left(K_{S}\right)\right]
$$

is a section of the quotient, a local isomorphism.

For more details, see [Mansfield et al. 2013].

Semenov-Tian-Shansky's twisted Poisson brackets. In this section we will assume that $\mathfrak{g}$ is semisimple and that $\langle\cdot, \cdot\rangle$ is a nondegenerate inner product in $\mathfrak{g}$ that allows us to identify $\mathfrak{g}$ and $\mathfrak{g}^{*}$ (a multiple of the one generated by the Killing form). Denote by $E_{i, j}$ the matrix with 0 s everywhere except for the $(i, j)$ entry, where it has a 1 . Since we are assuming that $G \subset \mathrm{GL}(n, \mathbb{R})$, we can assume that, for example, the inner product is the trace of the product of matrices, so that $E_{i, j}^{*}=E_{j, i}$. The following definitions and descriptions are due to Drinfeld [1983].

Definition 2.9 (Poisson-Lie group). A Poisson-Lie group is a Lie group equipped with a Poisson bracket such that the multiplication map $G \times G \rightarrow G$ is a Poisson map, where we consider the manifold $G \times G$ with the product Poisson bracket.

Definition 2.10 (Lie bialgebra). Let $\mathfrak{g}$ be a Lie algebra such that $\mathfrak{g}^{*}$ also has a Lie algebra structure given by a bracket $[\cdot, \cdot]_{*}$. Let $\delta: \mathfrak{g} \rightarrow \Lambda^{2} \mathfrak{g}$ be the dual map to the 
dual Lie bracket, that is,

$$
\langle\delta(v),(\xi \wedge \eta)\rangle=\left\langle[\xi, \eta]_{*}, v\right\rangle
$$

for all $\xi, \eta \in \mathfrak{g}^{*}, v \in \mathfrak{g}$. Assume that $\delta$ is a one-cocycle, that is

$$
\delta([v, w])=[v \otimes \mathbf{1}+\mathbf{1} \otimes v, \delta(w)]-[w \otimes \mathbf{1}+\mathbf{1} \otimes w, \delta(v)]
$$

for all $v, w \in \mathfrak{g}$. Then $\left(\mathfrak{g}, \mathfrak{g}^{*}\right)$ is called a Lie bialgebra.

If $G$ is a Lie-Poisson group, the linearization of the Poisson bracket at the identity defines a Lie bracket in $\mathfrak{g}^{*}$. The map $\delta$ is called the cobracket. The inverse result (any Lie bialgebra corresponds to a Lie-Poisson group) is also true for connected and simply connected Lie groups, as shown in [Drinfeld 1983].

Definition 2.11 (admissible subgroup). Let $M$ be a Poisson manifold, $G$ a PoissonLie group and $G \times M \rightarrow M$ a Poisson action. A subgroup $H \subset G$ is called admissible if the space $C^{\infty}(M)^{H}$ of $H$-invariant functions on $M$ is a Poisson subalgebra of $C^{\infty}(M)$.

The following proposition describes admissible subgroups.

Proposition 2.12 [Semenov-Tian-Shansky 1985]. Let $\left(\mathfrak{g}, \mathfrak{g}^{*}\right)$ be the tangent Lie bialgebra of a Poisson Lie group $G$. A Lie subgroup $H \subset G$ with Lie algebra $\mathfrak{h} \subset \mathfrak{g}$ is admissible if $\mathfrak{h}^{0} \subset \mathfrak{g}^{*}$ is a Lie subalgebra, where $\mathfrak{h}^{0}$ is the annihilator of $\mathfrak{h}$.

We will now describe the Poisson brackets that will be at the center of our study.

Definition 2.13 (factorizable Lie bialgebras and $R$-matrices). A Lie bialgebra $\left(\mathfrak{g}, \mathfrak{g}^{*}\right)$ is called factorizable if the following two conditions hold:

(a) $\mathfrak{g}$ is equipped with an invariant bilinear form $\langle\cdot, \cdot\rangle$ so that $\mathfrak{g}^{*}$ can be identified with $\mathfrak{g}$ via $\xi \in \mathfrak{g}^{*} \rightarrow v_{\xi} \in g$ with $\xi(\cdot)=\left\langle v_{\xi}, \cdot\right\rangle$

(b) the Lie bracket on $\mathfrak{g}^{*} \cong \mathfrak{g}$ is given by

$$
[\xi, \eta]_{*}=\frac{1}{2}([R(\xi), \eta]+[\xi, R(\eta)]),
$$

where $R \in \operatorname{End}(\mathfrak{g})$ is a skew-symmetric operator satisfying the modified classical Yang-Baxter equation

$$
[R(\xi), R(\eta)]=R([R(\xi), \eta]+[\xi, R(\eta)])-[\xi, \eta] .
$$

$R$ is called a classical $R$-matrix. Let $r$ be the 2-tensor image of $R$ under the identification $\mathfrak{g} \otimes \mathfrak{g} \cong \mathfrak{g} \otimes \mathfrak{g}^{*} \cong \operatorname{End}(\mathfrak{g})$. That is,

$$
r(\xi \wedge \eta)=\langle\xi, R(\eta)\rangle .
$$

The tensor $r$ is often referred to as the $R$-matrix also. 
The simplest example of an $R$-matrix is as follows: assume that $\mathfrak{g}$ has a splitting of the form $\mathfrak{g}=\mathfrak{g}_{+} \oplus \mathfrak{h}_{0} \oplus \mathfrak{g}_{-}$, where $\mathfrak{g}_{+}$and $\mathfrak{g}_{-}$are subalgebras dual of each other and where $\mathfrak{h}_{0}$ is commutative (for example, $\mathfrak{h}_{0}$ could be the Cartan subalgebra). Then it is well-known that the map $R: \mathfrak{g} \rightarrow \mathfrak{g}$ given by

$$
R\left(\xi_{+}+\xi_{0}+\xi_{-}\right)=\frac{1}{2}\left(\xi_{+}-\xi_{-}\right)
$$

defines a classical $R$-matrix.

Given a Poisson Lie group $G$ and its associated factorizable Lie bialgebra $\left(\mathfrak{g}, \mathfrak{g}^{*}\right)$, we can define an induced Poisson structure on $G^{N}$, as explained in [Semenov-TianShansky 1985]. Indeed, we equip $\mathfrak{g}^{N}=\bigoplus_{N} \mathfrak{g}$ with a nondegenerate inner product given by

$$
\langle X, Y\rangle=\sum_{k=1}^{N}\left\langle X_{k}, Y_{k}\right\rangle
$$

and we extend $R \in \operatorname{End}(\mathfrak{g})$ to $R \in \operatorname{End}\left(\mathfrak{g}^{N}\right)$ using $R\left(\left(X_{S}\right)\right)=\left(R\left(X_{S}\right)\right)$. Then $G^{N}$ is a Poisson Lie-group (with the product Poisson structure) and $\left(\mathfrak{g}^{N}, \mathfrak{g}_{R}^{N}\right)$ is its factorizable Lie bialgebra, where $\mathfrak{g}_{R}$ denotes $\mathfrak{g}$ with Lie bracket (9). Note that we are abusing notation, using $\langle\cdot, \cdot\rangle$ and $R$ to denote both the inner product and the $R$-matrix in $\mathfrak{g}$ and $\mathfrak{g}^{N}$. We will point out the difference only when it is not clear from the context and notation.

Definition 2.14 (left and right gradients). Let $\mathscr{F}: G^{N} \rightarrow \mathbb{R}$ be a differentiable function. We define the left gradient of $\mathscr{F}$ at $L=\left(L_{S}\right) \in G^{N}$ as the element of $\mathfrak{g}^{N}$ denoted by $\nabla \mathscr{F}(L)=\left(\nabla_{s} \mathscr{F}(L)\right)$, with $\nabla_{s} \mathscr{F}(L)$ satisfying

$$
\left.\frac{d}{d \epsilon}\right|_{\epsilon=0} \mathscr{F}\left(\left(\exp \left(\epsilon \xi_{s}\right) L_{s}\right)\right)=\left\langle\nabla_{s} \mathscr{F}(L), \xi_{s}\right\rangle
$$

for all $s$ and any $\xi=\left(\xi_{s}\right) \in \mathfrak{g}^{N}$.

Analogously, we define the right gradient of $\mathscr{F}$ at $L$ as the element of $\mathfrak{g}^{N}$ denoted by $\nabla^{\prime} \mathscr{F}(L)=\left(\nabla_{S}^{\prime} \mathscr{F}(L)\right)$, with $\nabla_{S}^{\prime} \mathscr{F}(L)$ satisfying

$$
\left.\frac{d}{d \epsilon}\right|_{\epsilon=0} \mathscr{F}\left(\left(L_{s} \exp \left(\epsilon \xi_{s}\right)\right)\right)=\left\langle\nabla_{s}^{\prime} \mathscr{F}(L), \xi_{s}\right\rangle
$$

for all $s$ and any $\xi=\left(\xi_{s}\right) \in \mathfrak{g}^{N}$. Clearly

$$
\nabla_{s}^{\prime} \mathscr{F}(L)=L_{s}^{-1} \nabla_{s} \mathscr{F}(L) L_{s} .
$$

If $r$ is given as in (10) for some $R$-matrix $R$, the Poisson structure in $G^{N}$ given by the formula

$$
\left\{\mathscr{F}_{,} \mathscr{G}_{S}(L)=\sum_{s=1}^{N} \hat{r}\left(\nabla_{s} \mathscr{F} \wedge \nabla_{s} \mathscr{G}\right)-\sum_{s=1}^{N} \hat{r}\left(\nabla_{s}^{\prime} \mathscr{F} \wedge \nabla_{S}^{\prime} \mathscr{G}\right)\right.
$$


is called the Sklyanin bracket. Now, given a factorizable Lie bialgebra, SemenovTian-Shansky [1985] defined what is called a twisted Poisson structure on $G^{N}$. Here we will give the definition of this structure, and we refer the reader to the same reference for explanations on how to obtain it and to [Frenkel et al. 1998, Theorem 1] for the explicit formula.

Let $\mathscr{F}, \varphi: G^{N} \rightarrow \mathbb{R}$ be two functions. Let $\tau$ be the shift operator $\tau\left(X_{S}\right)=X_{S+1}$. We define the $\tau$-twisted Poisson bracket as

$$
\begin{aligned}
\{\mathscr{F}, \mathscr{G}\}(L) & =\sum_{s=1}^{N} r\left(\nabla_{s} \mathscr{F} \wedge \nabla_{s} \varphi\right)+\sum_{s=1}^{N} r\left(\nabla_{s}^{\prime} \mathscr{F} \wedge \nabla_{s}^{\prime} \varphi\right) \\
& -\sum_{s=1}^{N}(\tau \otimes \mathrm{id})(r)\left(\nabla_{s}^{\prime} \mathscr{F} \otimes \nabla_{s} \varphi\right)+\sum_{s=1}^{N}(\tau \otimes \mathrm{id})(r)\left(\nabla_{s}^{\prime} \varphi \otimes \nabla_{s} \mathscr{F}\right) .
\end{aligned}
$$

In [Frenkel et al. 1998; Semenov-Tian-Shansky 1985] it was proved that not only is this a Poisson bracket but the gauge action of $G^{N}$ on itself, that is, the action $G^{N} \times G^{N} \rightarrow G^{N}$ given by

$$
\left(L_{s}\right) \rightarrow\left(g_{s+1} L_{s} g_{s}^{-1}\right),
$$

is a Poisson map and the gauge orbits are Poisson submanifolds. This is the relevant bracket to our study of polygon evolutions.

\section{A Hamiltonian bracket on the moduli space of twisted polygons in parabolic manifolds}

Let $G$ be a semisimple group and $\mathfrak{g}$ its Lie algebra. Assume $\mathfrak{g}$ has a gradation of the form

$$
\mathfrak{g}=\mathfrak{g}_{1} \oplus \mathfrak{g}_{0} \oplus \mathfrak{g}_{-1},
$$

where $\mathfrak{g}_{1}$ and $\mathfrak{g}_{-1}$ are dual to each other with respect to an adjoint-invariant inner product. Let $G_{i}$ be the subgroup of $G$ with Lie algebra $\mathfrak{g}_{i}$, and $P \subset G$ the parabolic subgroup of $G$ with Lie algebra $\mathfrak{p}=\mathfrak{g}_{1} \oplus \mathfrak{g}_{0}$.

Consider the space of polygons in the homogeneous manifold $M=G / P$. In this section we will show that under some assumptions, (14), defined in $G^{N}$, can be reduced to the quotient $G^{N} / P^{N}$ to define a Poisson structure on the space of Maurer-Cartan matrices associated to polygons in $M$, and hence on the space of invariants as shown in Theorem 2.8.

Before we go into our main theorem, we will recall some known facts about the action of $G$ on $G / P$ when $\mathfrak{g}$ is a $|1|$-graded algebra as in (16). The following descriptions can be found, for example, in [Ochiai 1970]. Let $G_{1}$ and $G_{-1}$ be the 
connected Lie subgroups of $G$ corresponding to $\mathfrak{g}_{1}$ and $\mathfrak{g}_{-1}$, respectively. We define $G_{0}$ to be the normalizer of $\mathfrak{g}_{0}$ in $P$, that is, $G_{0}=\left\{a \in P \mid \operatorname{Ad}(a)\left(\mathfrak{g}_{0}\right)=\mathfrak{g}_{0}\right\}$.

Proposition 3.1. The exponential mappings $\exp : \mathfrak{g}_{1} \rightarrow G_{1}$ and $\exp : \mathfrak{g}_{-1} \rightarrow G_{-1}$ are bijective. Furthermore, $G_{0}$ is also the normalizer of $\mathfrak{g}_{-1}$ in $P$ and $P$ is the semidirect product of $G_{0}$ and $G_{1}$.

The subgroup $G_{0}$ is called the linear isotropy subgroup of the semisimple homogeneous space $G / P$ and it is clearly locally bijective, via the exponential map, to $\mathfrak{g}_{0}$. Perhaps a more important description for this paper is the following well-known result. It can be obtained from [Ochiai 1970], although here it is simplified for a clearer exposition.

Proposition 3.2. Let $G \times M \rightarrow M$ be the action of $G$ on $M=G / P$ given by left multiplication on class representatives. Let $G_{i}$ and $\mathfrak{g}_{i}$ be given as above, $i=1,0,-1$. Then the infinitesimal action of $\mathfrak{g}_{-1}$ is constant in $x$, the one of $\mathfrak{g}_{0}$ is linear in $x$ and the one of $\mathfrak{g}_{1}$ is quadratic in $x$.

Next, assume that $\mathfrak{g}$ can be endowed with two different splittings: the original parabolic gradation (16), and a splitting of the form

$$
\mathfrak{g}=\mathfrak{g}_{-} \oplus \mathfrak{h} \oplus \mathfrak{g}_{+},
$$

where $\mathfrak{h}_{0}$ is commutative and $\mathfrak{g}_{-}$and $\mathfrak{g}_{+}$are dual to each other. Assume also that this splitting can be chosen so that $\mathfrak{g}_{1} \subset \mathfrak{g}_{+}, \mathfrak{g}_{-1} \subset \mathfrak{g}_{-}$and $\mathfrak{h} \subset \mathfrak{g}_{0}$, while $\mathfrak{g}_{0}$ will have, in general, intersection with all $\mathfrak{g}_{+}, \mathfrak{h}$ and $\mathfrak{g}_{-}$.

Remark 3.3. This assumption is not too restrictive. For example, in the complex case, given a simple Lie algebra (a semisimple one will be the sum of its simple terms) one can always find two gradations related as above, a pair per root with weight equal to 1 . One way to find the gradations is as follows: ${ }^{1}$ Let $\mathfrak{h}$ be a choice of Cartan subalgebra and $\Delta=\left\{\alpha_{r}\right\}_{r=1}^{\ell}$ a simple root system associated to $\mathfrak{h}$. Let $\Phi^{+}$be the set of positive roots and $\Phi^{-}$the set of negative roots. Let $\lambda \in \Delta$ have weight 1 and let $\Phi_{1}=\left\{\alpha \in \Phi^{+}\right.$with $\lambda$ in its linear expansion $\}$ and $\Phi_{-1}$ the negative analogue. Define $\mathfrak{g}_{1}=\bigoplus_{\alpha \in \Phi_{1}} \mathfrak{g}_{\alpha}$ and $\mathfrak{g}_{-1}=\bigoplus_{\alpha \in \Phi_{-1}} \mathfrak{g}_{\alpha}$. Let $\mathfrak{g}_{0}$ be the sum of the root spaces associated to the remaining roots (the ones that do not contain $\lambda$ ). We see that $\mathfrak{g}_{1}$ is commutative since $\lambda$ cannot appear in any linear expansion with a coefficient higher than one, and if $\alpha, \beta \in \Phi_{1}$, then the coefficient of $\alpha+\beta$ would be 2 . Likewise with $\Phi_{-1}$. The second gradation is simply given by $\mathfrak{g}_{+}=\bigoplus_{\alpha \in \Phi^{+}} \mathfrak{g}_{\alpha}, \mathfrak{g}_{-}=\bigoplus_{\alpha \in \Phi^{-}} \mathfrak{g}_{\alpha}$ and $\mathfrak{h}$, ensuring that $\mathfrak{g}_{1} \subset \mathfrak{g}_{+}$and $\mathfrak{g}_{-1} \subset \mathfrak{g}_{-}$.

Not all algebras have such roots. The ones that do are: $A_{r}$ (with $r$ different choices of roots), $B_{r}$ (one choice), $C_{r}$ (one choice), $D_{r}$ (3 choices), $E_{6}$ (two

\footnotetext{
${ }^{1}$ The author is very grateful to Professor Georgia Benkart for the description and discussions on this matter.
} 
choices), $E_{7}$ (one choice). In the case of a simple real Lie algebra, Kobayashi and Nagano [1964; 1965] described all semisimple real Lie algebras with gradations (16) as direct sums of simple ones belonging to the following list:

(1) $\mathfrak{g}=\mathfrak{s l}(p+q, \mathbb{R})$ with $\mathfrak{g}_{0}=\mathfrak{s l}(p, \mathbb{R}) \oplus \mathfrak{s l}(q, \mathbb{R}) \oplus \mathbb{R}$;

(2) $\mathfrak{g}=\mathfrak{s o}(n, n)$ with $\mathfrak{g}_{0}=\mathfrak{g l}(n, \mathbb{R})$;

(3) $\mathfrak{g}=\mathfrak{s o}(p+1, q+1)$ with $\mathfrak{g}_{0}=\mathfrak{s o}(p+q) \oplus \mathbb{R}$;

(4) $\mathfrak{g}=\mathfrak{s p}(2 n, \mathbb{R})$ with $\mathfrak{g}_{0}=\mathfrak{g l}(n, \mathbb{R})$;

(5) $\mathfrak{g}=E_{6}^{1}$ with $\mathfrak{g}_{0}=\mathfrak{s o}(5,5) \oplus \mathbb{R}$;

(6) $\mathfrak{g}=E_{7}^{1}$ with $\mathfrak{g}_{0}=E_{6}^{1} \oplus \mathbb{R}$.

Using their representations, one can see that the standard finest gradation inherited from $\mathfrak{g l}(n, \mathbb{R})$ with $n=p+q$ will work as gradation (17) for (1); case (2) is very similar to (4), which we will describe in detail in our last section, while cases (3), (5) and (6) are not clear to us. As the reader can see, some of the exceptional cases satisfy our assumptions.

We are now ready for our main theorem.

Theorem 3.4. Assume $G$ and $\mathfrak{g}$ are as above. The twisted Poisson structure (14) defined on $G^{N}$, with $r$ associated to (17) as in (11), is locally reducible to the quotient $G^{N} / P^{N}$, and the reduced bracket coincides with the reduction of the Sklyanin bracket (13) with tensor

$$
\hat{r}(\xi, \eta)=\left\langle\xi_{-1}, \eta_{1}\right\rangle
$$

where $\xi_{-1}$ and $\eta_{1}$ correspond to the parabolic gradation (16) defining $M$.

Notice that $\hat{r}$ is not an $R$-matrix and hence the Sklyanin bracket is not Poisson before reduction.

Proof. The proof is similar to the one for $\mathbb{R} \mathbb{P}^{n}$ that appeared in [Marí Beffa and Wang 2013], with some differences. From Theorem 2.8, the quotient is locally a manifold, and as explained in [Semenov-Tian-Shansky 1985] the gauge action is a Poisson action for the twisted bracket, whose symplectic leaves are gauge orbits. Therefore, using the same reasoning as the one used in [Marí Beffa and Wang 2013, Theorem 5.5] we conclude that the bracket can be reduced whenever $P$ is admissible (see Definition 2.11). According to Proposition 2.12, this is true whenever $\mathfrak{p}^{0}=\mathfrak{g}_{1}$ is a Lie subalgebra of $\mathfrak{g}^{*}$, and this is the only condition we need to check to prove the first part of the theorem.

The Lie bracket in $\mathfrak{g}^{*}$ is defined by the linearization of the twisted Poisson bracket at the identity $e \in G$. That is,

$$
\left[d_{e} \phi, d_{e} \varphi\right]_{*}=d_{e}\{\phi, \varphi\} \in \mathfrak{g}^{*} .
$$


Since $\mathfrak{p}^{0}=\mathfrak{g}_{1}$, we will look for functions $\varphi_{s}^{i}$ such that $d_{e} \varphi_{s}^{i}$ generate $\mathfrak{g}_{1}$.

First of all, we can locally identify $M$ with the section represented by $G_{-1}$ through a map $x \mapsto \ell(x) \in G_{-1}$. Let $\varphi: U \subset M \rightarrow \mathbb{R}^{n}$ be local coordinates around $o$ defined as follows: choose coordinates for $G_{-1}$ given by the exponential map composed with linear coordinates in $\mathfrak{g}_{-1}$, and define $\varphi(\ell(x))=\varphi(x)$. Assume $\varphi^{i}$ are the components of $\varphi$; that is, if $w_{i}$ are generators of $\mathfrak{g}_{-1}$, then $\ell(x)=\Pi_{i} \exp \left(\alpha_{i} w_{i}\right)$ and $\varphi^{i}(x)=\alpha_{i}, i=1, \ldots, n$ (recall that $G_{-1}$ is commutative). Now, let $L \in G^{N}$ be close enough to $e \in G^{N}$ so that $L=\left(L_{s}\right)$ can be factored as $L_{s}=L_{-1}^{s} L_{0}^{s} L_{1}^{s}$ with $L_{i}^{s} \in G_{i}$, according to the gradation (16). We choose $x_{s} \in U \subset M$ such that $L_{-1}^{s}=$ $\ell\left(x_{s}\right)$, and define $\varphi^{i}(L)=\left(\varphi^{i}\left(L_{s}\right)\right)=\left(\varphi^{i}\left(L_{-1}^{s}\right)\right)=\left(\varphi^{i}\left(\ell\left(x_{s}\right)\right)\right)=\left(\varphi^{i}\left(x_{s}\right)\right)$. Since $d_{e} \varphi^{i}$ is in the dual of the tangent to $M$ at the identity (which we can identify with $\mathfrak{g}_{-1}$, with dual equal to $\left.\mathfrak{g}_{1}\right)$ and $\varphi$ are coordinates, the elements $d_{e} \varphi^{i}, i=1, \ldots, n$, must generate $\mathfrak{g}_{1}=\mathfrak{p}^{0}$. Now we only need to check that if $\{\cdot, \cdot\}$ is the quotient bracket in (14), then

$$
\left[d_{e} \varphi^{i}, d_{e} \varphi^{j}\right]_{*}=d_{e}\left\{\varphi^{i}, \varphi^{j}\right\} \in \mathfrak{p}^{0}=\mathfrak{g}_{1} .
$$

This will imply that $P$ is admissible.

Identify $M^{N}$ with the section represented by $G_{-1}^{N}$ via the map

$$
\left(x_{s}\right) \rightarrow\left(\ell\left(x_{s}\right)\right) \in G_{-1}^{N} .
$$

Then the action of $G^{N}$ on $M^{N}$ is uniquely determined by the relation

$$
g_{s} \ell\left(x_{s}\right)=\ell\left(g_{s} \cdot x_{s}\right) p_{s}
$$

for some $p_{s} \in P$. Let $\xi_{s} \in \mathfrak{g}$ and $V_{s}=\exp \left(\epsilon \xi_{s}\right)$. As before, assume $L_{s}=L_{-1}^{s} L_{0}^{s} L_{1}^{s}$ with $L_{-1}^{s}=\ell\left(x_{s}\right)$ for some $x_{s} \in M$. Let $V_{s}=\exp \left(\epsilon \xi_{s}\right)$. Using (18), we obtain

$$
\varphi\left(V_{s} L_{s}\right)=\varphi\left(V_{s} L_{-1}^{s}\right)=\varphi\left(V_{s} \ell\left(x_{s}\right)\right)=\varphi\left(\ell\left(V_{s} \cdot x_{s}\right)\right)=\varphi\left(V_{s} \cdot x_{s}\right) .
$$

(1) If $\xi_{s} \in \mathfrak{g}_{-1}$ and given that the infinitesimal action of $\mathfrak{g}_{-1}$ on $M$ is constant, we have that

$$
\left.\frac{d}{d \epsilon}\right|_{\epsilon=0} \varphi\left(V_{s} L_{s}\right)=\left\langle\nabla_{s} \varphi\left(L_{s}\right), \xi_{s}\right\rangle
$$

is constant in $L_{s}$. That is to say, if $\nabla_{s} \varphi\left(L_{s}\right)$ splits according to the parabolic gradation (16), then its $\mathfrak{g}_{1}$ component is constant for any $L_{s}$ and for all $s$.

(2) If $\xi \in \mathfrak{g}_{0}$, then $\varphi\left(V_{s} L_{s}\right)$ is again $\varphi\left(V_{s} \cdot x_{s}\right)$ as above. The infinitesimal action is now linear, and hence $\nabla_{s} \varphi\left(L_{s}\right)$ has a $\mathfrak{g}_{0}$-component that is linear in $L_{-1}^{s}=$ $\ell\left(x_{s}\right)$, for all $s$. This will vanish at $x_{s}=0$, or what is the same, at $L_{s}=e$.

(3) If $\xi \in \mathfrak{g}_{1}$, the infinitesimal action will be quadratic, and hence $\nabla_{s} \varphi\left(L_{s}\right)$ will have a $\mathfrak{g}_{-1}$ component that is quadratic in $L_{-1}^{s}=\ell\left(x_{s}\right)$, for all $s$. Thus, it vanishes at $x_{s}=0$ or $L_{s}=e$. 
We now calculate $d_{e}\left\{\varphi^{i}, \varphi^{j}\right\}$ where $\{\cdot, \cdot\}$ is the twisted bracket (14) with the $r$-matrix given by (11). We want to show that $d_{e}\left\{\varphi^{i}, \varphi^{j}\right\} \in \mathfrak{p}^{0}$ and so we need to show that $\left.\frac{d}{d \epsilon}\right|_{\epsilon=0}\left\{\varphi_{s}^{i}, \varphi_{s}^{j}\right\}\left(e^{\epsilon \xi}\right)=0$ whenever $\xi \in \mathfrak{p}=\mathfrak{g}_{1} \oplus \mathfrak{g}_{0}$.

Given that $\left(d_{e} \varphi_{s}^{i}\right)_{-1}=0$ and $\left(\nabla \varphi_{s}^{i}(L)\right)_{-1}$ is quadratic in $\ell\left(x_{s}\right)$ (with $\ell(o)=e$ ) we can conclude that $\left.\frac{d}{d \epsilon}\right|_{\epsilon=0}\left(\nabla \varphi_{S}^{i}\left(e^{\epsilon \xi}\right)\right)_{-1}=0$.

Also, $\nabla^{\prime} \varphi_{s}^{i}\left(e^{\epsilon \xi}\right)=e^{-\epsilon \xi} \nabla \varphi_{s}^{i}\left(e^{\epsilon \xi}\right) e^{\epsilon \xi}$, and therefore

$$
\left.\frac{d}{d \epsilon}\right|_{\epsilon=0} \nabla^{\prime} \varphi_{S}^{i}\left(e^{\epsilon \xi}\right)=\left[d_{e} \varphi_{s}^{i}, \xi_{s}\right]+\left.\frac{d}{d \epsilon}\right|_{\epsilon=0} \nabla \varphi_{S}^{i}\left(e^{\epsilon \xi}\right) .
$$

Since $d_{e} \varphi_{s}^{i} \in \mathfrak{g}_{1}$, whenever $\xi \in \mathfrak{p}$ we have that $\left[d_{e} \varphi_{s}^{i}, \xi_{s}\right] \in \mathfrak{p}$ and hence

$$
\left.\frac{d}{d \epsilon}\right|_{\epsilon=0}\left(\nabla^{\prime} \varphi_{s}^{i}\left(e^{\epsilon \xi}\right)\right)_{-1}=0 .
$$

Furthermore, $\left(d_{e} \varphi_{s}^{i}\right)_{0}=0$ also. Finally, we split

$$
\left\langle\nabla_{+} \varphi_{s}^{i}, \nabla_{-} \varphi_{s}^{i}\right\rangle=\left\langle\nabla_{1} \varphi_{s}^{i}, \nabla_{-1} \varphi_{s}^{i}\right\rangle+\left\langle\nabla_{+}^{0} \varphi_{s}^{i}, \nabla_{-}^{0} \varphi_{s}^{i}\right\rangle,
$$

where $\nabla_{+}^{0} \varphi_{s}^{i}$ and $\nabla_{-}^{0} \varphi_{s}^{i}$ are the components of $\nabla_{+} \varphi_{s}^{i}$ and $\nabla_{+} \varphi_{s}^{i}$ in $\mathfrak{g}_{0}$. Substituting this splitting in the definition of the twisted bracket and going over each one of its terms, we get that they all vanish, $\left.\frac{d}{d \epsilon}\right|_{\epsilon=0}\left\{\varphi_{s}^{i}, \varphi_{s}^{j}\right\}\left(e^{\epsilon \xi}\right)=0$, and hence $\mathfrak{p}^{0}$ is a subalgebra of $\mathfrak{g}^{*}$.

We now look at the second assertion of the theorem. The reduced bracket is calculated as follows: let $f, h: \mathscr{K} \rightarrow \mathbb{R}$ be two functions on the quotient space $\mathscr{K}=$ $U^{N} / P^{N}$, where $P^{N}$ is acting on the open set $U^{N} \subset M^{N}$ by gauge transformations. Consider two extensions of $f, h$ to $U^{N}$, call them $\mathscr{F}$ and $\mathscr{H}$, constant on the gauge leaves of $P$. That means

$$
\mathscr{F}\left(p_{s+1} K_{s} p_{s}^{-1}\right)=\mathscr{F}\left(K_{S}\right)=f\left(\boldsymbol{k}_{s}\right)
$$

for any $p_{s} \in P$, where $\boldsymbol{k}_{s}$ are coordinates for $K_{s}$ (i.e., a generating system of invariants defined by $\left.K_{S}\right)$. Choosing $p_{s}=\exp \left(\epsilon \xi_{s}\right), \xi_{s} \in \mathfrak{p}$ and differentiating, we get that

$$
\sum_{s=1}^{N}\left\langle-\nabla_{s}^{\prime} \mathscr{F}^{-1} \nabla_{s} \mathscr{F}, \xi_{s}\right\rangle=0
$$

That is,

$$
-\nabla^{\prime} \mathscr{F}+\tau^{-1} \nabla \mathscr{F} \in\left(\mathfrak{p}^{0}\right)^{N}=\mathfrak{g}_{1}^{N} .
$$

Likewise for $\mathscr{H}$. The reduced bracket is then defined as

$$
\{f, h\}_{\text {inv }}(\boldsymbol{k})=\{\mathscr{F}, \mathscr{H}\}(K) .
$$


We now use this description to finish the proof. Since $\tau \nabla^{\prime} \mathscr{F}-\nabla \mathscr{F} \in\left(\mathfrak{p}^{0}\right)^{N}=\mathfrak{g}_{1}^{N}$ and $\mathfrak{g}_{-} \subset \mathfrak{g}_{-1} \oplus \mathfrak{g}_{0}$, we have that $\tau\left(\nabla^{\prime} \mathscr{F}\right)_{-}=(\nabla \mathscr{F})_{-}$, and from this the reduced Poisson bracket can be expressed as

$$
\begin{aligned}
\{f, h\}(\boldsymbol{k})= & \frac{1}{2}\left(\left\langle(\nabla \mathscr{F})_{-},(\nabla \mathscr{H})_{+}\right\rangle-\left\langle(\nabla \mathscr{F})_{+},(\nabla \mathscr{H})_{-}\right\rangle\right. \\
& \left.+\left\langle\left(\nabla^{\prime} \mathscr{F}\right)_{-},\left(\nabla^{\prime} \mathscr{H}\right)_{+}\right\rangle-\left\langle\left(\nabla^{\prime} \mathscr{F}\right)_{+},\left(\nabla^{\prime} \mathscr{H}\right)_{-}\right\rangle\right) \\
& -\left\langle\tau\left(\nabla^{\prime} \mathscr{F}\right)_{-},(\nabla \mathscr{H})_{+}\right\rangle+\left\langle\tau\left(\nabla^{\prime} \mathscr{H}\right)_{-},(\nabla \mathscr{F})_{+}\right\rangle \\
= & \frac{1}{2}\left(-\left\langle(\nabla \mathscr{F})_{-},(\nabla \mathscr{H})_{+}\right\rangle+\left\langle(\nabla \mathscr{F})_{+},(\nabla \mathscr{H})_{-}\right\rangle\right. \\
& \left.+\left\langle\left(\nabla^{\prime} \mathscr{F}\right)_{-},\left(\nabla^{\prime} \mathscr{H}\right)_{+}\right\rangle-\left\langle\left(\nabla^{\prime} \mathscr{F}\right)_{+},\left(\nabla^{\prime} \mathscr{H}\right)_{-}\right\rangle\right) \\
& -\frac{1}{2}\left(-\left\langle(\nabla \mathscr{F})_{-},(\nabla \mathscr{H})_{+}\right\rangle+\left\langle(\nabla \mathscr{F})_{+},(\nabla \mathscr{H})_{-}\right\rangle\right. \\
& \left.+\left\langle\left(\nabla^{\prime} \mathscr{F}\right)_{-},\left(\nabla^{\prime} \mathscr{H}\right)_{+}\right\rangle-\left\langle\left(\nabla^{\prime} \mathscr{F}\right)_{+},\left(\nabla^{\prime} \mathscr{H}\right)_{-}\right\rangle\right) \\
= & \frac{1}{2}\left\langle(\nabla \mathscr{H})_{-},(\nabla \mathscr{F})_{+}-\tau\left(\nabla^{\prime} \mathscr{F}\right)_{+}\right\rangle-\frac{1}{2}\left\langle(\nabla \mathscr{F})_{-},(\nabla \mathscr{H})_{+}-\tau\left(\nabla^{\prime} \mathscr{H}\right)_{+}\right\rangle .
\end{aligned}
$$

Since $\mathfrak{g}_{1} \subset \mathfrak{g}_{+}$, this is equal to

$$
\frac{1}{2}\left\langle(\nabla \mathscr{H})_{-1},(\nabla \mathscr{F})_{1}-\tau\left(\nabla^{\prime} \mathscr{F}\right)_{1}\right\rangle-\frac{1}{2}\left\langle(\nabla \mathscr{F})_{-1},(\nabla \mathscr{H})_{1}-\tau\left(\nabla^{\prime} \mathscr{H}\right)_{1}\right\rangle,
$$

and from this we can go back to

$$
\begin{aligned}
- & \frac{1}{2}\left(-\left\langle(\nabla \mathscr{F})_{-1},(\nabla \mathscr{H})_{1}\right\rangle+\left\langle(\nabla \mathscr{F})_{1},(\nabla \mathscr{H})_{-1}\right\rangle\right. \\
& \left.+\left\langle\left(\nabla^{\prime} \mathscr{F}\right)_{-1},\left(\nabla^{\prime} \mathscr{H}\right)_{1}\right\rangle-\left\langle\left(\nabla^{\prime} \mathscr{F}\right)_{1},\left(\nabla^{\prime} \mathscr{H}\right)_{-1}\right\rangle\right),
\end{aligned}
$$

which coincides with the evaluation of (13) defined by the parabolic gradation (16) on the extensions $\mathscr{F}$ and $\mathscr{H}$.

\section{Polygon evolutions inducing a Hamiltonian evolution on invariants}

In this section we will study which invariant evolutions of polygons induce an evolution on $\boldsymbol{k}$ which is Hamiltonian with respect to the reduced bracket we described in our previous section. In particular, we will link the invariant vector $\boldsymbol{v}_{S}$ describing the evolution (4), to the gradient of the Hamiltonian $f$ determining the evolution of the invariants. The relation is simple and straightforward and we will show that any Hamiltonian flow on the invariants is induced by a polygon evolution.

First of all, recall that if $\left(x_{s}\right)$ evolves under (4), then the evolution of the MaurerCartan invariants is given by (6), where $N=\rho_{t} \rho^{-1} \in \mathfrak{g}^{N}$ satisfies the condition

$$
\left(N_{S}\right)_{-1}=-d \varsigma(o) v_{S} .
$$

Lemma 4.1. Let $h$ be a function of the invariants $\boldsymbol{k}$, and let $\mathscr{H}$ be an extension of $h$ constant on the gauge orbits of $P$. Assume that, for a fixed function $f$, 


$$
\sum_{s=1}^{N}\left\langle\nabla_{s} \mathscr{H},\left(K_{s}\right)_{t} K_{s}^{-1}\right\rangle=\{f, h\}_{\text {inv }}(\boldsymbol{k}) \text { for any function } h .
$$

Then $K_{t} K^{-1}$ defines $a\{\cdot, \cdot\}_{\mathrm{inv}}$-Hamiltonian evolution on the coordinates $\boldsymbol{k}$, with Hamiltonian $f$.

Proof. Let $\boldsymbol{k}=\left(\boldsymbol{k}_{s}\right)$ and $\boldsymbol{k}_{s}=\left(\boldsymbol{k}_{s}^{i}\right)$ be coordinates for $K$ (we write $\left.K_{s}=K_{s}(\boldsymbol{k})\right)$, and assume $x$ evolves according to (4). The evolution induced on $K_{s}$ (through $\boldsymbol{k}$ ) is given by the relation

$$
\left(K_{S}\right)_{t} K_{s}^{-1}=\sum_{r=1}^{N} \sum_{i=1}^{n}\left(\boldsymbol{k}_{r}^{i}\right)_{t} \frac{\partial K_{s}}{\partial \boldsymbol{k}_{r}^{i}} K_{s}^{-1} .
$$

On the other hand, let $h$ be a function of $\boldsymbol{k}$ and $\mathscr{H}$ an extension constant on the leaves of $P$. If $Z_{s}=K_{s}\left(\boldsymbol{k}_{r}^{i}(\epsilon)\right) K_{s}^{-1}\left(\boldsymbol{k}_{r}^{i}\right)$, with $\boldsymbol{k}_{r}^{i}(0)=\boldsymbol{k}_{r}^{i}$ and $\left.\frac{d}{d \epsilon}\right|_{\epsilon=0} \boldsymbol{k}_{r}^{i}(0)=v_{r}^{i}$, we have

$$
\left.\frac{d}{d \epsilon}\right|_{\epsilon=0} \mathscr{H}\left(Z_{S} K_{S}\right)=\sum_{s}\left\langle\nabla_{S} \mathscr{H}(K),\left.\frac{d}{d \epsilon}\right|_{\epsilon=0} Z_{S}\right\rangle
$$

on the one side, while on the other side

$$
\left.\frac{d}{d \epsilon}\right|_{\epsilon=0} \mathscr{H}\left(Z_{S} K_{S}\right)=\left.\frac{d}{d \epsilon}\right|_{\epsilon=0} \mathscr{H}\left(K_{S}\left(\boldsymbol{k}_{r}^{i}(\epsilon)\right)\right)=\left.\frac{d}{d \epsilon}\right|_{\epsilon=0} h\left(\boldsymbol{k}_{r}^{i}(\epsilon)\right)=\sum_{r=1}^{N} \sum_{i=1}^{n} v_{r}^{i} \frac{\partial h}{\partial \boldsymbol{k}_{r}^{i}} .
$$

We further see that

$$
\left.\frac{d}{d \epsilon}\right|_{\epsilon=0} Z_{S}=\sum_{r=1}^{N} \sum_{i=1}^{n} v_{r}^{i} \frac{\partial K}{\partial \boldsymbol{k}_{r}^{i}} K^{-1} .
$$

Comparing the two sides of (22), which must be equal for any values of $v_{r}^{i}$, we arrive at

Finally, assume that

$$
\frac{\partial h}{\partial \boldsymbol{k}_{r}^{i}}=\sum_{s}\left\langle\nabla_{s} \mathscr{H}(K), \frac{\partial K_{s}}{\partial \boldsymbol{k}_{r}^{i}} K_{s}^{-1}\right\rangle .
$$

$$
\sum_{s=1}^{N}\left\langle\nabla_{s} \mathscr{H},\left(K_{s}\right)_{t} K_{s}^{-1}\right\rangle=\{f, h\}_{\text {inv }}(\boldsymbol{k})
$$

for any $h$. Then

$$
\sum_{s=1}^{N} \sum_{r=1}^{N} \sum_{i=1}^{n}\left\langle\left(\boldsymbol{k}_{r}^{i}\right)_{t} \frac{\partial K_{s}}{\partial \boldsymbol{k}_{r}^{i}} K_{s}^{-1}, \nabla_{s} \mathscr{H}\right\rangle=\sum_{s} \sum_{r, i}\left(\boldsymbol{k}_{r}^{i}\right)_{t} \frac{\partial h}{\partial k_{r}^{i}}=\{f, h\}_{\text {inv }}(\boldsymbol{k})
$$

for any $h$, and hence, by definition, $\boldsymbol{k}$ evolves via a Hamiltonian evolution, with Hamiltonian function $f$. 
Theorem 4.2. Let $\left(x_{s}\right)$ evolve using an evolution of the form (4), for some invariant vector $\boldsymbol{v}_{s}$, and let $\varsigma$ be a section such that $\rho_{s}=\rho_{s}^{P} \varsigma\left(x_{s}\right)^{-1}, \rho_{s}^{P} \in P$, for any $s$. Assume that there exits a function $f(\boldsymbol{k})$, with extension $\mathscr{F}_{\mathrm{F}}$ constant on the gauge orbits of $\mathscr{P}$, and such that

$$
d \varsigma(o) v_{S}=\tau^{-1}\left(\nabla_{S} \mathscr{F}\right)_{-1} .
$$

The evolution induced on $\boldsymbol{k}$ by (4) is Hamiltonian with respect to $\{\cdot, \cdot\}_{\mathrm{inv}}$, with Hamiltonian $f$.

Proof. Using (6) and (21), we have that, on the one hand

$$
\sum_{s=1}^{N}\left\langle\left(K_{s}\right)_{t} K_{s}^{-1}, \nabla_{s} \mathscr{H}\right\rangle=\sum_{s=1}^{N}\left\langle N_{s+1}-K_{s} N_{s} K_{s}^{-1}, \nabla_{s} \mathscr{H}\right\rangle,
$$

and on the other hand

$$
\{f, h\}_{\text {inv }}(\boldsymbol{k})=\frac{1}{2} \sum_{s=1}^{N}\left\langle\left(\nabla_{s} \mathscr{H}\right)_{-1},\left(\nabla_{s} \mathscr{F}-\tau \nabla_{s}^{\prime} \mathscr{F}\right)_{1}\right\rangle-\left\langle\left(\nabla_{s} \mathscr{F}\right)_{-1},\left(\nabla_{s} \mathscr{H}-\tau \nabla_{s}^{\prime} \mathscr{H}\right)_{1}\right\rangle .
$$

Now, since $\nabla_{s} \mathscr{F}-\tau \nabla_{s}^{\prime} \mathscr{F}_{\mathcal{F}} \in \mathfrak{g}_{1}$, and $\mathfrak{g}_{-1}$ is the dual of $\mathfrak{g}_{1}$, we have

$$
\begin{aligned}
\left\langle\left(\nabla_{s} \mathscr{H}\right)_{-1},\left(\nabla_{s} \mathscr{F}-\tau \nabla_{s}^{\prime} \mathscr{F}\right)_{1}\right\rangle & =\left\langle\nabla_{s} \mathscr{H}, \nabla_{s} \mathscr{F}-\tau \nabla_{s}^{\prime} \mathscr{F}\right\rangle \\
& =\left\langle\nabla_{s} \mathscr{H}, \nabla_{s} \mathscr{F}\right\rangle-\left\langle\nabla_{s} \mathscr{H}, \tau \nabla_{s}^{\prime} \mathscr{F}\right\rangle .
\end{aligned}
$$

Also, since $\langle\cdot, \cdot\rangle$ is invariant under the adjoint action and under the shift operator, $\sum_{s}\left\langle\nabla_{s} \mathscr{H}, \nabla_{s} \mathscr{F}\right\rangle=\sum_{s}\left\langle\tau \nabla_{s}^{\prime} \mathscr{H}, \tau \nabla_{s}^{\prime} \mathscr{F}\right\rangle$. Substituting this in our calculations we get

$$
\begin{aligned}
\sum_{s}\left\langle\left(\nabla_{s} \mathscr{H}\right)_{-1},\left(\nabla_{s} \mathscr{F}-\tau \nabla_{s}^{\prime} \mathscr{F}\right)_{1}\right\rangle & =\sum_{s}\left\langle\tau \nabla_{s}^{\prime} \mathscr{H}, \tau \nabla_{s}^{\prime} \mathscr{F}\right\rangle-\left\langle\nabla_{s} \mathscr{H}, \tau \nabla_{s}^{\prime} \mathscr{F}\right\rangle \\
& =\sum_{s}\left\langle\tau \nabla_{s}^{\prime} \mathscr{H}-\nabla_{s} \mathscr{H}, \tau \nabla_{s}^{\prime} \mathscr{F}\right\rangle \\
& =\sum_{s}\left\langle\left(\tau \nabla_{s}^{\prime} \mathscr{H}-\nabla_{s} \mathscr{H}\right)_{1},\left(\tau \nabla_{s}^{\prime} \mathscr{F}\right)_{-1}\right\rangle \\
& =\sum_{s}\left\langle\left(\tau \nabla_{s}^{\prime} \mathscr{H}-\nabla_{s} \mathscr{H}\right)_{1},\left(\nabla_{s} \mathscr{F}\right)_{-1}\right\rangle,
\end{aligned}
$$

where we have used that $\left(\nabla_{s} \mathscr{F}\right)_{-1}=\left(\tau \nabla_{s}^{\prime} \mathscr{F}\right)_{-1}$ since $\nabla_{s} \mathscr{F}-\tau \nabla_{s}^{\prime} \mathscr{F} \in \mathfrak{g}_{1}$. Therefore

$$
\{f, h\}_{\text {inv }}(\boldsymbol{k})=-\sum_{s=1}^{N}\left\langle\left(\nabla_{s} \mathscr{F}\right)_{-1},\left(\nabla_{s} \mathscr{H}-\tau \nabla_{s}^{\prime} \mathscr{H}\right)_{1} .\right.
$$

Back to (24). Since

$$
\sum_{s}\left\langle K_{s} N_{s} K_{s}^{-1}, \nabla_{s} \mathscr{H}\right\rangle=\sum_{s}\left\langle N_{s}, K_{s}^{-1} \nabla_{s} \mathscr{H} K_{s}\right\rangle=\sum_{s}\left\langle\tau N_{s}, \tau\left(K_{s}^{-1} \nabla_{s} \mathscr{H} K_{s}\right)\right\rangle,
$$


we have

$$
\begin{aligned}
\sum_{s=1}^{N}\left\langle N_{s+1}-K_{s} N_{s} K_{s}^{-1}, \nabla_{s} \mathscr{H}\right\rangle & =\sum_{s=1}^{N}\left\langle\tau N_{s}, \nabla_{s} \mathscr{H}-\tau\left(K_{s}^{-1} \nabla_{s} \mathscr{H} K_{s}\right)\right\rangle \\
& =\sum_{s=1}^{N}\left\langle\tau N_{s}, \nabla_{s} \mathscr{H}-\tau \nabla_{s}^{\prime} \mathscr{H}\right\rangle \\
& =\sum_{s=1}^{N}\left(\left\langle\tau N_{s}\right)_{-1},\left(\nabla_{s} \mathscr{H}-\tau \nabla_{s}^{\prime} \mathscr{H}\right)_{1}\right\rangle .
\end{aligned}
$$

But, if $\tau \varsigma(o) v_{S}=\nabla_{-1} \mathscr{F}\left(\right.$ and since $\left(N_{S}\right)_{-1}=-d \varsigma(o) v_{S}$ by $\left.(7)\right)$, we get $\left(\tau N_{S}\right)_{-1}=$ $-(\nabla \mathscr{F})_{-1}$, and hence

$$
\sum_{s=1}^{N}\left\langle\left(K_{s}\right)_{t} K_{s}^{-1}, \nabla_{s} \mathscr{H}\right\rangle=-\sum_{s=1}^{N}\left\langle\left(\nabla_{s} \mathscr{F}\right)_{-1},\left(\nabla_{s} \mathscr{H}-\tau \nabla_{s}^{\prime} \mathscr{H}\right)_{1}\right\rangle=\{f, h\}_{\text {inv }}(\boldsymbol{k}) .
$$

Using our previous lemma, we conclude the proof.

Remark 4.3. In all examples we can think of the values of $\left(N_{s}\right)_{-1}=-d \varsigma(o) v_{s}$ and condition (4) as determining $N_{s}$ uniquely. This means that if $x_{s}$ induces an evolution on $\boldsymbol{k}$ which is Hamiltonian with respect to $\{\cdot, \cdot\}_{\text {inv }}$ with Hamiltonian $f$, then necessarily $\tau\left(N_{S}\right)_{-1}=-\left(\nabla_{S} \mathscr{F}\right)_{-1}$, since this choice induces the same evolution and $N_{S}$ is unique given those determining values. Hence, assuming that $N$ is uniquely determined by $\left(N_{S}\right)_{-1}, s=1, \ldots, N$, and (4), the converse of the theorem is also true.

\section{The Lagrangian Grassmannian example: the Lagrangian Schwarzian difference and Volterra evolutions}

In this section we apply the previous construction to the case of the Lagrangian Grassmannian. In this example $G=\operatorname{Sp}(2 n)$ and the parabolic gradation of the algebra is given by

$$
\left(\begin{array}{ll}
\mathbf{0} & \mathbf{0} \\
Z & \mathbf{0}
\end{array}\right) \in \mathfrak{g}_{1}, \quad\left(\begin{array}{cc}
A & \mathbf{0} \\
\mathbf{0} & -A^{T}
\end{array}\right) \in \mathfrak{g}_{0}, \quad\left(\begin{array}{ll}
\mathbf{0} & Y \\
\mathbf{0} & \mathbf{0}
\end{array}\right) \in \mathfrak{g}_{-1},
$$

where $\mathbf{0}$ is the zero $n \times n$ block, $Z$ and $Y$ are symmetric matrices, and $A$ is a general $n \times n$ matrix. Here $\mathfrak{p}=\mathfrak{g}_{0} \oplus \mathfrak{g}_{1}$. The associated local factorization of the group is given by

$$
g=\left(\begin{array}{cc}
I & \mathbf{0} \\
\widehat{S} & I
\end{array}\right)\left(\begin{array}{cc}
\Theta & \mathbf{0} \\
\mathbf{0} & \Theta^{-T}
\end{array}\right)\left(\begin{array}{cc}
I & S \\
\mathbf{0} & I
\end{array}\right) \in G_{1} G_{0} G_{-1}
$$


with $\Theta \in \operatorname{GL}(n, \mathbb{R}), \hat{S}$ and $S$ symmetric. Also, $P=G_{1} G_{0}$ and $G / P$ is the Lagrangian Grassmannian. For a more geometric definition, consider $n$ vectors defining a given $n$-dimensional subspace $x$ of $\mathbb{R}^{2 n}$. We can find $n$ such vectors so that when placed as columns in a matrix, the matrix will look like

$$
\left(\begin{array}{l}
u \\
I
\end{array}\right) \text {. }
$$

If the subspace is Lagrangian, $u$ will be symmetric. We identify this subspace with the matrix

$$
\varsigma(x)=\left(\begin{array}{ll}
I & u \\
0 & I
\end{array}\right) \in G_{-1},
$$

which defines a section of the quotient $G / P$.

The second gradation (17) is given by

$$
\left(\begin{array}{cc}
A_{L} & \mathbf{0} \\
C & -A_{L}^{T}
\end{array}\right) \in \mathfrak{g}_{+}, \quad\left(\begin{array}{cc}
d & \mathbf{0} \\
\mathbf{0} & -d
\end{array}\right) \in \mathfrak{h}, \quad\left(\begin{array}{cc}
A_{U} & B \\
\mathbf{0} & -A_{U}^{T}
\end{array}\right) \in \mathfrak{g}_{-},
$$

where $A_{L}$ is strictly lower triangular, $A_{U}$ is strictly upper triangular, and $d$ is diagonal. One can readily see that $\mathfrak{g}_{+}, \mathfrak{g}_{-}$and $\mathfrak{h}$ are all subalgebras of $\mathfrak{g}$. Also clearly $\mathfrak{h}$ is commutative, $\mathfrak{g}_{1} \subset \mathfrak{g}_{+}, \mathfrak{g}_{-1} \subset \mathfrak{g}_{-}$and $\mathfrak{h} \subset \mathfrak{g}_{0}$, so that we can apply Theorem 3.4 to obtain a Poisson bracket on the moduli space of Lagrangian Grassmannian polygons. This structure is, in general, very complicated. What we want to do in this section is to show that some of the invariants of Lagrangian polygons behave in familiar and interesting ways under selected evolutions. For this we will go into details, constructing explicitly the invariants and their evolutions. We will then restrict the reduced bracket further to a submanifold generated by these special invariants.

A moving frame along Lagrangian Grassmannian polygons. Let $g$ be factored as in (26). If we identify $M$ with symmetric matrices $u$, using the section (27), and given that the action of $\operatorname{Sp}(2 n)$ on $M$ is determined by (18), we can write the action explicitly as

$$
g \cdot u=\Theta(u+S)\left(\Theta^{-T}+\widehat{S} \Theta(u+S)\right)^{-1} .
$$

Assume we factor our right moving frame $\rho=\left(\rho_{S}\right) \in \operatorname{Sp}(2 n)^{N}$ according to (26) as

$$
\rho_{s}=\left(\begin{array}{cc}
I & \mathbf{0} \\
\widehat{S}_{s} & I
\end{array}\right)\left(\begin{array}{cc}
\Theta_{s} & \mathbf{0} \\
\mathbf{0} & \Theta_{s}^{-T}
\end{array}\right)\left(\begin{array}{cc}
I & S_{s} \\
\mathbf{0} & I
\end{array}\right) .
$$

If we define transverse sections as in (1) through the normalizations

$$
\rho_{s} \cdot u_{s}=\mathbf{0}, \quad \rho_{s} \cdot u_{s-1}=-I, \quad \rho_{s} \cdot u_{s+1}=I,
$$


we obtain the equations

$$
\begin{aligned}
u_{s}+S_{s} & =\mathbf{0}, \\
\Theta_{s}\left(u_{s+1}+S_{s}\right) & =\Theta_{s}^{-T}+\widehat{S}_{s} \Theta_{s}\left(u_{s+1}+S_{s}\right), \\
\Theta_{s}\left(u_{s-1}+S_{s}\right) & =-\left(\Theta_{s}^{-T}+\widehat{S}_{s} \Theta_{s}\left(u_{s-1}+S_{s}\right)\right) .
\end{aligned}
$$

These can be solved for

$$
S_{S}=-u_{s}, \quad \hat{S}_{s}=I-\Theta_{s}^{-T}\left(u_{s+1}-u_{s}\right)^{-1} \Theta_{s}^{-1}
$$

and

$$
\Theta_{s}^{T} \Theta_{s}=\frac{1}{2}\left(\left(u_{s+1}-u_{s}\right)^{-1}+\left(u_{s}-u_{s-1}\right)^{-1}\right)=U_{s}^{-1} .
$$

Equation (30) determines $\Theta$ completely up to an orthogonal factor, assuming that $U_{s}^{-1}=\frac{1}{2}\left(\left(u_{s+1}-u_{s}\right)^{-1}+\left(u_{s}-u_{s-1}\right)^{-1}\right)$ is positive definite. In fact, we have $\Theta_{s}=\theta_{s} U_{s}^{-1 / 2}$, where $\theta_{s} \in \mathrm{O}(n)$ and $U_{s}^{-1 / 2}$ is a square root of a symmetric matrix as defined in [Ovsienko 1993], unique up to the action of the orthogonal group. That is, $U_{s}^{-T / 2} U_{s}^{-1 / 2}=U_{s}^{-1}$.

To determine the last factor $\theta_{s}$, and with it the rest of the moving frame, we need to choose one more normalization, thus completing the definition of the transverse section. Let's choose $\rho_{s} \cdot u_{s+2}$ to be diagonal. After substituting all the values we have already found we get

$$
\rho_{s} \cdot u_{s+2}=\left(I+\Theta^{-T}\left(\left(u_{s+2}-u_{s}\right)^{-1}-\left(u_{s+1}-u_{s}\right)^{-1}\right) \Theta^{-1}\right)^{-1} .
$$

Definition 5.1 (Lagrangian Schwarzian difference). Given a generic polygon of Lagrangian planes $u_{s}$, we define $S(u)=\left(S_{S}(u)\right)$ to be

$$
\begin{aligned}
S_{s}(u) & =U_{s}^{-1 / 2}\left(U_{s}^{-1}+\left(u_{s+2}-u_{s}\right)^{-1}-\left(u_{s+1}-u_{s}\right)^{-1}\right)^{-1} U_{s}^{-T / 2} \\
& =U_{s}^{-1 / 2}\left[\left(u_{s+2}-u_{s}\right)^{-1}-\frac{1}{2}\left(u_{s+1}-u_{s}\right)^{-1}+\frac{1}{2}\left(u_{s}-u_{s-1}\right)^{-1}\right]^{-1} U_{s}^{-T / 2}
\end{aligned}
$$

and we call it the Lagrangian Schwarzian difference of $u$, where $U_{s}$ is as in (30).

This definition is the discrete analogue to the Lagrangian Schwarzian derivative defined in [Ovsienko 1993] for curves of Lagrangian planes. In fact, if we denote $u(s+k)=\gamma(x+k \epsilon)$, a long but standard calculation shows that the continuous limit of $S_{S}(u)$ is indeed a multiple of the Lagrangian Schwarzian derivative defined in the same reference. Now, in order to diagonalize $\rho_{s} \cdot u_{s+2}$ we need to choose $\theta_{s}$ to be an element of the orthogonal group that diagonalizes the symmetric matrix $S_{S}(u)$. If we call

$$
\widetilde{D}_{s}=\theta_{s} S_{S}(u) \theta_{s}^{T}
$$


then $\rho_{s} \cdot u_{s+2}=\widetilde{D}$ will be diagonal. These normalization choices describe transverse sections as in (1), and they determine the moving frame $\rho$ uniquely. From now on we will denote $D_{s}=I-\widetilde{D}_{s}^{-1}$, and hence $I_{s+2}^{s}=\left(I+D_{s}\right)^{-1}$.

Maurer-Cartan invariants and their evolutions. Once we have determined a moving frame, we would like to describe the right Maurer-Cartan matrix associated to it. To do this we will use the recursion equations (3)

$$
K_{s} \cdot I_{k}^{s}=I_{k}^{s+1} \text {. }
$$

Using the choices $I_{s}^{s}=\mathbf{0}, I_{s+1}^{s}=I, I_{s-1}^{s}=-I$ and $I_{s+2}^{s}=\left(I+D_{s}\right)^{-1}$, we select the equations

$$
K_{s} \cdot \mathbf{0}=-I, \quad K_{s} \cdot I=\mathbf{0}, \quad K_{s} \cdot I_{s+2}^{s}=I,
$$

as those determining $K$. Assume that $K_{s}$ factorizes as

$$
K_{s}=\left(\begin{array}{cc}
I & \mathbf{0} \\
K_{s, 1} & I
\end{array}\right)\left(\begin{array}{cc}
K_{s, 0} & \mathbf{0} \\
\mathbf{0} & \left(K_{s, 0}\right)^{-T}
\end{array}\right)\left(\begin{array}{cc}
I & K_{s,-1} \\
\mathbf{0} & I
\end{array}\right)
$$

Straightforward calculations using formula (29) show that the three recursion equations (32) determine the values

$$
K_{s,-1}=-I, \quad K_{s, 0}^{T} K_{s, 0}=-\frac{1}{2} D_{s}^{-1}, \quad K_{s, 1}=K_{s, 0}^{-T} K_{s, 0}^{-1}-I .
$$

Assuming that $D_{s}$ is negative definite, we obtain the solutions

$$
K_{s,-1}=-I, \quad K_{s, 0}=\widehat{K}_{s, 0} \widehat{D}_{s}, \quad K_{s, 1}=-\left(I+2 \widehat{K}_{s, 0} D_{s} \hat{K}_{s, 0}^{T}\right),
$$

where

$$
\widehat{D}_{s}=\frac{1}{\sqrt{2}}\left(-D_{s}\right)^{-1 / 2}, \quad \widehat{K}_{s, 0} \in \mathrm{O}(n) .
$$

Remark 5.2. the negative definite condition imposed on $D_{s}$ can be removed by merely choosing different normalizations. Indeed, if we choose arbitrary values for $I_{r}^{S}$, the relations between the different invariants determined by equations (32) become

$$
K_{s, 0}^{T}\left(\left(I_{s+1}^{s}\right)^{-1}-\left(I_{s-1}^{s}\right)^{-1}\right) K_{s, 0}=-\left(I_{s+1}^{s}\right)^{-2} D_{s}^{-1} .
$$

Thus, if $D_{s}$ is positive definite, we could choose $I_{s+1}^{s}=-I$ and $I_{s-1}^{s}=I$ instead. We can also change the sign of the different entries in $I$, depending on the sign of the different eigenvalues of $S(u)$. For simplicity we will keep the choices above.

The following theorem summarizes our findings. 
Theorem 5.3. There exists a right moving frame along polygons of Lagrangian subspaces such that its associated Maurer-Cartan matrix is of the form

$$
K_{s}=\left(\begin{array}{cc}
I & \mathbf{0} \\
-\left(I+2 \hat{K}_{s, 0} D_{s} \hat{K}_{s, 0}^{T}\right) & I
\end{array}\right)\left(\begin{array}{cc}
\hat{K}_{s, 0} \widehat{D}_{s} & \mathbf{0} \\
\mathbf{0} & \widehat{K}_{s, 0}^{T} \widehat{D}_{s}^{-1}
\end{array}\right)\left(\begin{array}{cc}
I & -I \\
\mathbf{0} & I
\end{array}\right)
$$

where $\widehat{D}_{s}$ is given as in (34). The entries of $D_{s}$ and $\widehat{K}_{s, 0}$ functionally generate all invariants of Lagrangian polygons.

Next we turn to the study of invariant evolutions of Lagrangian polygons (that is, those for which $\operatorname{Sp}(2 n)$ takes solutions to solutions) and the equations they induce on the invariants. Assume $\left(u_{s}(t)\right)$, with $u_{s}(t)$ symmetric, represents a family of polygons of Lagrangian planes, and assume it is a solution of an invariant evolution. According to Theorem 2.6, the equation can be written in terms of our moving frame. Since the linearization at $o$ of the action (29) is given by

$$
v \mapsto \Theta v \Theta^{T}
$$

and having in mind that the $G_{0}$ factor of $\rho_{s}^{-1}$ is $U_{s}^{1 / 2} \theta_{s}^{T}$, from (4) we conclude that any invariant evolution can be written as

$$
\left(u_{S}\right)_{t}=U_{s}^{1 / 2} \theta_{s}^{T} \boldsymbol{v}_{s} \theta_{S} U_{s}^{T / 2}
$$

for symmetric matrices $\boldsymbol{v}_{s}$ depending on the entries of $\left(D_{r}\right)$ and $\left(\hat{K}_{r, 0}\right)$, and where $\theta_{s}$ diagonalizes the Lagrangian Schwarzian difference of the flow. From now on we will assume that $D_{s}=\operatorname{diag}\left(d_{i}^{s}\right)$, with $d_{i}^{s} \neq d_{j}^{s}$ for all $i \neq j$.

Theorem 5.4. Let $\boldsymbol{v}_{s}$ be diagonal, and assume the initial condition $u_{s}(0)$ satisfies $\widehat{K}_{s, 0}=I$. Then $\widehat{K}_{s, 0}=I$ is preserved by the flow (36) and whenever $\boldsymbol{v}_{s}=$ $-\frac{1}{2}\left(1+\tau^{-1}\right) D_{s} \nabla_{s} f$ for some Hamiltonian function $f, D_{s}$ satisfies a Hamiltonian equation with respect to the Poisson structure

$$
\mathscr{P}=\sum_{s} D_{s}\left(D_{s} \tau^{-1}-D_{s} \tau+2\left(\tau^{-1}-\tau\right)+\tau^{-1} D_{s}-\tau D_{s}+\tau^{-1} D_{s} \tau^{-1}-\tau D_{s} \tau\right) D_{s} .
$$

with Hamiltonian $f$. Assume $\boldsymbol{v}_{s}=-I$ (and hence $\nabla_{s} f=D_{s}^{-1}$ ). Then, as polygons evolve following

$$
\left(u_{s}\right)_{t}=2\left(\left(u_{s}-u_{s+1}\right)^{-1}-\left(u_{s}-u_{s-1}\right)^{-1}\right)^{-1},
$$

the eigenvalues of the Lagrangian Schwarzian difference evolve following a decoupled system of Volterra equations.

Proof. From now on, and to avoid cluttering, we will drop the subindex $s$ and will only use it if needed to avoid confusion. Thus, $N_{s+1}$ will become $\tau N, N_{s}$ will become $N, \widehat{D}_{s}$ will become $\widehat{D}$, and so on. 
We will use Theorem 2.7. Consider the section $\varsigma: M \rightarrow G_{-1}$ given by

$$
\varsigma(u)=\left(\begin{array}{ll}
I & u \\
\mathbf{0} & I
\end{array}\right) \text {. }
$$

It satisfies $\rho=\rho^{P} \varsigma(u)^{-1}$ with $\rho^{P} \in P$. Theorem 2.7 tells us that $N=\rho_{t} \rho^{-1}$ must be of the form

$$
N=\left(\begin{array}{cc}
N_{0} & -v \\
N_{1} & -N_{0}^{T}
\end{array}\right)
$$

for some $N_{0} \in \mathfrak{g l}(n), N_{1}$ symmetric and for some symmetric matrix $\boldsymbol{v}$ depending on the invariants. A straightforward calculation shows that if $K$ is as in (35), and if $\widehat{K}_{0}=I$, then

$K N K^{-1}=\left(\begin{array}{cc}I & \mathbf{0} \\ K_{1} & I\end{array}\right)\left(\begin{array}{cc}\widehat{D}\left(N_{0}-N_{1}\right) \widehat{D}^{-1} & \widehat{D}\left(N_{0}+N_{0}^{T}-N_{1}-\boldsymbol{v}\right) \hat{D} \\ \hat{D}^{-1} N_{1} \widehat{D}^{-1} & \widehat{D}^{-1}\left(N_{1}-N_{0}^{T}\right) \widehat{D}\end{array}\right)\left(\begin{array}{cc}I & \mathbf{0} \\ -K_{1} & I\end{array}\right)$, where $K_{1}=-(I+2 D)$. To simplify formulas we will conjugate (4) by $\left(\begin{array}{cc}I & 0 \\ -K_{1} & I\end{array}\right)$. Direct, although longer, calculations show that if $\widehat{K}_{0}=I$

$\left(\begin{array}{cc}I & \mathbf{0} \\ -K_{1} & I\end{array}\right) K_{t} K^{-1}\left(\begin{array}{cc}I & \mathbf{0} \\ K_{1} & I\end{array}\right)=\left(\begin{array}{cc}\left(\widehat{K}_{0}\right)_{t}-\frac{1}{2} D^{-1} D_{t} & \mathbf{0} \\ 2\left(-D_{t}+D\left(\widehat{K}_{0}\right)_{t}-\left(\widehat{K}_{0}\right)_{t} D\right) & \left(\widehat{K}_{0}\right)_{t}+\frac{1}{2} D^{-1} D_{t}\end{array}\right)$,

where we have used the relationship

$$
\widehat{D}^{-1}(\widehat{D})_{t}=-\frac{1}{2} D^{-1} D_{t}
$$

Also

$$
\begin{aligned}
& \left(\begin{array}{cc}
I & \mathbf{0} \\
-K_{1} & I
\end{array}\right)\left(\begin{array}{cc}
\tau N_{0} & -\tau \boldsymbol{v} \\
\tau N_{1} & -\tau N_{0}^{T}
\end{array}\right)\left(\begin{array}{cc}
I & \mathbf{0} \\
K_{1} & I
\end{array}\right)= \\
& \left(\begin{array}{cc}
\tau N_{0}+\tau \boldsymbol{v}(I+2 D) & -\tau \boldsymbol{v} \\
\tau N_{1}+\tau N_{0}+\tau N_{0}^{T}+2 D \tau N_{0}+2 T N_{0}^{T} D+(I+2 D)^{2} \tau \boldsymbol{v} & -\tau N_{0}^{T}-(I+2 D) \tau \boldsymbol{v}
\end{array}\right) .
\end{aligned}
$$

Substituting these values in the conjugation of 2.7 by $\left(\begin{array}{cc}I & 0 \\ -K_{1} & I\end{array}\right)$, and equating the different entries, we arrive at the equations

$$
\begin{aligned}
& \tau \boldsymbol{v}=\widehat{D}\left(\boldsymbol{v}+N_{1}-N_{0}-N_{0}^{T}\right) \hat{D}, \\
& D^{-1} D_{t}=\widehat{D}\left(N_{0}-N_{1}\right) \widehat{D}^{-1}+\widehat{D}^{-1}\left(N_{0}^{T}-N_{1}\right) \widehat{D}-2(I+2 D) \tau \boldsymbol{v}-\tau N_{0}-\tau N_{0}^{T}, \\
& 2\left(\widehat{K}_{0}\right)_{t}=\tau N_{0}^{T}-\tau N_{0}+\widehat{D}\left(N_{0}-N_{1}\right) \widehat{D}^{-1}+\widehat{D}^{-1}\left(N_{1}-N_{0}^{T}\right) \widehat{D}, \\
& 2\left(D\left(\widehat{K}_{0}\right)_{t}-\left(\widehat{K}_{0}\right)_{t} D-D_{t}\right)=\tau N_{1}+\tau N_{0}+\tau N_{0}^{T}+2\left(D \tau N_{0}+\tau N_{0}^{T} D\right) \\
& \quad+(I+2 D)^{2} \tau \boldsymbol{v}-\widehat{D}^{-1} N_{1} \widehat{D}^{-1} .
\end{aligned}
$$

The last three equations result in a compatibility condition that can be obtained as follows: we use the second equation and we multiply once on the left and once 
on the right by $D$, thus obtaining two equations. We substitute the sum of these two equations in place of $2 D_{t}$ in the last equation, and use the third equation to substitute $\left(\widehat{K}_{0}\right)_{t}$. After some straightforward work we obtain

$$
\tau N_{1}+\tau N_{0}+\tau N_{0}^{T}=\hat{D}^{-1}\left(N_{0}+N_{0}^{T}-N_{1}\right) \hat{D}^{-1}-\tau \boldsymbol{v}\left(I-4 D^{2}\right) .
$$

We now use the first equation, and we get

$$
\tau N_{1}=-(\tau(D \tau \boldsymbol{v})+\tau \boldsymbol{v}+D \boldsymbol{v}) .
$$

From this, if $\boldsymbol{v}$ is diagonal, so is $N_{1}$. The first equation implies that $N_{0}+N_{0}^{T}$ is also diagonal. Back to the second equation, we see that $\widehat{D} N_{0} \widehat{D}^{-1}+\widehat{D}^{-1} N_{0}^{T} \widehat{D}$ is once more diagonal, which with our assumption $d_{i} \neq d_{j}$ implies that $N_{0}$ is diagonal. If $N_{0}$ and $N_{1}$ are diagonal, the third equation tells us that $\left(\hat{K}_{0}\right)_{t}=0$, proving the first assertion of the theorem.

We can now find $D_{t}$. Using (38) and the first equation we have

$$
2 N_{0}=\left(D \tau-\tau^{-1} D\right) \boldsymbol{v},
$$

and substituting everything into the second equation we get

$$
D^{-1} D_{t}=\left(D-\tau D \tau+\tau^{-1} D-D \tau+2-2 \tau\right) \boldsymbol{v} .
$$

Finally, if we substitute $v=\frac{1}{2}\left(1+\tau^{-1}\right) D \nabla f$, we have

$$
D_{t}=\frac{1}{2} D\left(D \tau^{-1}-D \tau+2\left(\tau^{-1}-\tau\right)+\tau^{-1} D-\tau D+\tau^{-1} D \tau^{-1}-\tau D \tau\right) D \nabla f
$$

which is a Hamiltonian equation with respect to the Hamiltonian structure

$$
\mathscr{P}=D\left(D \tau^{-1}-D \tau+2\left(\tau^{-1}-\tau\right)+\tau^{-1} D-\tau D+\tau^{-1} D \tau^{-1}-\tau D \tau\right) D .
$$

This is the second Hamiltonian structure for the Volterra equation

$$
D_{t}=D\left(\tau-\tau^{-1}\right) D
$$

(see [Khanizadeh et al. 2013]), which we obtain whenever $\boldsymbol{v}=I$ and $\nabla f=D^{-1}$. Adopting subindices again and using (36) and (30), if $\boldsymbol{v}_{s}=I$, the corresponding evolution for $u_{s}$ is given by

$$
\left(u_{s}\right)_{t}=U_{s}^{-1 / 2} U_{s}^{-T / 2}=U_{s}^{-1}=2\left(\left(u_{s+1}-u_{s}\right)^{-1}+\left(u_{s}-u_{s-1}\right)^{-1}\right)^{-1}
$$

concluding the proof of the theorem.

Of course, we did not guess the relation

$$
\boldsymbol{v}=\frac{1}{2}\left(1+\tau^{-1}\right) D \nabla f
$$


it was given to us by the general reduction process and the compatibility condition (23). We will describe the reduction next for our particular example and see how we arrived to relation (41).

The reduced bracket and the double reduction to $D$. Before we prove that the reduced bracket found in the previous section is further reducible to $D$, we will describe the reduced bracket itself in a little more detail. Once more we are dropping the subindex to avoid cluttering. As explained in (21), to calculate the reduction one considers two functions $f$ and $h$ defined on the invariants (coordinates) generating $D$ and $\widehat{K}_{0}$. Let us denote these invariants by $d=\left(d_{i}\right)$ and $k_{i, j}, i<j$. Let us also denote by $\delta_{1} f$ the diagonal matrix $\delta_{1} f=\operatorname{diag} \partial f / \partial d_{i}$, and by $\delta_{0} f$ a skew symmetric matrix generated by $\partial f / \partial k_{i, j}$. (The precise form of $\delta_{0} f$ will become clear along the process, therefore we will postpone the description until relevant.)

Set

$$
\nabla \mathscr{F}=\left(\begin{array}{cc}
F_{0} & F_{-1} \\
F_{1} & -F_{0}^{T}
\end{array}\right)
$$

and let us split $F_{0}$ as

$$
F_{0}=F_{0}^{\sigma}+F_{0}^{\sigma k}+F_{0}^{d},
$$

where $\sigma$ indicates the symmetric diagonal free components, $\sigma k$ is the skewsymmetric component, and $d$ the diagonal. Likewise for $F_{-1}$ and $F_{1}$ (clearly $\left.F_{-1}^{\sigma k}=F_{1}^{\sigma k}=0\right)$. Let us denote by $K\left(D, \widehat{K}_{0}\right)$ the family of matrices (35), and consider the element of $\operatorname{Sp}(2 n)^{N}$

$$
Z(\epsilon)=K\left(D+\epsilon V, \widehat{K}_{0}\right) K\left(D, \widehat{K}_{0}\right)^{-1},
$$

where $V$ is an arbitrary diagonal matrix. Let us call $v$ the diagonal of $V$, written as a vector. On the one hand, direct calculations show that when $\hat{K}_{0}=I$,

$$
\left.\frac{d}{d \epsilon}\right|_{\epsilon=0} Z(\epsilon)=\left(\begin{array}{cc}
-\frac{1}{2} D^{-1} V & \mathbf{0} \\
D^{-1} V & \frac{1}{2} D^{-1} V
\end{array}\right) .
$$

On the other hand $Z(\epsilon) K=K\left(D+\epsilon V, \widehat{K}_{0}\right)$ and since $\mathscr{F}$ is an extension of $f$

$$
F(Z(\epsilon) K)=F\left(K\left(D+\epsilon V, \widehat{K}_{0}\right)\right)=f\left(d+\epsilon v, k_{i, j}\right) .
$$

Differentiating with respect to $\epsilon$,

$$
\left\langle\nabla F,\left(\begin{array}{cc}
-\frac{1}{2} D^{-1} V & \mathbf{0} \\
D^{-1} V & \frac{1}{2} D^{-1} V
\end{array}\right)\right\rangle=\left\langle D^{-1}\left(F_{-1}^{d}-F_{0}^{d}\right), V\right\rangle=\left\langle\delta_{1} f, V\right\rangle .
$$

This is true for any value of $V$, and therefore

$$
F_{-1}^{d}-F_{0}^{d}=D \delta_{1} f \text {. }
$$


Likewise, we can choose $Z(\epsilon)$ such that

$$
F(Z(\epsilon) K)=F\left(D, \widehat{K}_{0}(\epsilon)\right)=f\left(d, k_{i, j}(\epsilon)\right)
$$

with $k_{i, j}(0)=k_{i, j}$ and $\left.\frac{d}{d \epsilon}\right|_{\epsilon=0} k_{i, j}(\epsilon)=w_{i, j}$. Indeed $Z(\epsilon)=K\left(D, \widehat{K}_{0}(\epsilon)\right) K^{-1}$, with $\widehat{K}_{0}(\epsilon)$ chosen so that $\widehat{K}_{0}(0)=\widehat{K}_{0}$ and $\left.\hat{K}_{0}^{-1} \frac{d}{d \epsilon}\right|_{\epsilon=0} \widehat{K}_{0}=W, W=\left(w_{i, j}\right)$. (One can be more specific and create $Z(\epsilon)$ using the exponential function, but any such family can be used and the precise form is not relevant.)

Differentiating with respect to $\epsilon$, we have

$$
\left\langle\nabla F,\left(\begin{array}{cc}
W & \mathbf{0} \\
\mathbf{0} & W
\end{array}\right)\right\rangle=\left\langle 2 F_{0}, W\right\rangle=\left\langle\delta_{0} f, W\right\rangle,
$$

and hence $F_{0}^{-}=\frac{1}{2} \delta_{0} f$. We can now determine how the matrix $\delta_{0} f$ is created: it is defined as the skew-symmetric matrix such that

$$
\left.\frac{d}{d \epsilon}\right|_{\epsilon=0} f\left(d, k_{i, j}(\epsilon)\right)=\left\langle\delta_{0} f, W\right\rangle
$$

with $W=\left(w_{i, j}\right)$ (that is, $\left.\left(\delta_{0} f\right)_{i, j}=\partial f / \partial k_{j, i}\right)$. With this in mind, we proceed to our last theorem.

Theorem 5.5. The reduced Poisson bracket (21) can be further reduced to the submanifold $\widehat{K}_{0}=I$. When using the coordinates given by the invariants $D$, the resulting bracket is a decoupled system of Hamiltonian structures for the Volterra equation as in (37).

Proof. More precisely, what we will show is that if $f$ is independent of $k_{i, j}$ and $h$ is independent of $d_{i}$, at $\widehat{K}_{0}=I$ their reduced bracket vanishes, while the reduced bracket of two functions that only depend on $D$ is given by the second Volterra structure. Once again, and as explained in (21), if $f$ and $h$ are two functions depending on $D$ and $\widehat{K}_{0}$, their reduced bracket is defined as

$$
\{f, h\}_{\text {inv }}=\frac{1}{2}\left\langle(\nabla \mathscr{H})_{-1},(\nabla \mathscr{F})_{1}-\tau\left(\nabla^{\prime} \mathscr{F}\right)_{1}\right\rangle-\frac{1}{2}\left\langle(\nabla \mathscr{F})_{-1},(\nabla \mathscr{H})_{1}-\tau\left(\nabla^{\prime} \mathscr{H}\right)_{1}\right\rangle,
$$

where $\mathscr{F}$ and $\mathscr{H}$ are two extensions satisfying (20). Assume $\nabla \mathscr{F}$ is given as in (42) with the splitting (43). After conjugating $\nabla^{\prime} \mathscr{F}$ back to $\nabla \mathscr{F}$ and simplifying, condition (20) results in two equations at $\widehat{K}_{0}=I$, namely

$$
\begin{aligned}
\tau^{-1} F_{0} & =\hat{D}^{-1}\left(F_{0}-F_{-1} K_{1}\right) \hat{D}+\widehat{D}\left(F_{1}+K_{1} F_{0}+F_{0}^{T} K_{1}-K_{1} F_{-1} K_{1}\right) \hat{D} \\
\tau^{-1} F_{-1} & =\hat{D}\left(F_{-1}-F_{0}^{T}\right) \hat{D}^{-1}-\tau^{-1} F_{0} .
\end{aligned}
$$

Case 1: Assume that $f$ depends only on $D$. Then $F_{0}^{\sigma k}=\frac{1}{2} \delta_{0} f=\mathbf{0}$. Using (45),

$$
\mathbf{0}=\tau^{-1}\left(F_{0}-F_{0}^{T}\right)=\widehat{D}^{-1}\left(F_{0}-F_{-1} K_{1}\right) \hat{D}-\widehat{D}\left(F_{0}^{T}-K_{1} F_{-1}\right) \widehat{D}^{-1} .
$$


Using that $K_{1}=I-\widehat{D}^{-2}$, we get

$$
\mathbf{0}=\hat{D}^{-1}\left(F_{0}-F_{-1}\right) \hat{D}-\widehat{D}\left(F_{0}^{T}-F_{-1}\right) \hat{D}^{-1},
$$

which, under the condition $d_{i} \neq d_{j}$, implies

$$
F_{0}^{\sigma}=F_{-1}^{\sigma}
$$

Substituting this in (45) we get

$$
2 \tau F_{1}^{\sigma}=\widehat{D}^{-1} F_{-1}^{\sigma} \widehat{D}^{-1}-\widehat{D}^{-1} F_{-1}^{\sigma} \widehat{D}^{-1}=\mathbf{0}
$$

and, therefore,

$$
F_{-1}^{\sigma}=F_{0}^{\sigma}=\mathbf{0} .
$$

This implies that $F_{1}$ and $F_{0}$ are both diagonal. If we now look at (45) we clearly see that $F_{1}$ is also diagonal. These diagonals can easily be found from (45)-(46) and the relation $F_{-1}^{d}=D \delta_{1} f+F_{0}^{d}$. They are

$$
\begin{aligned}
F_{-1}^{d} & =\frac{1}{2}(\tau+I) D \delta_{1} f \\
F_{0}^{d} & =\frac{1}{2}(\tau-I) D \delta_{1} f \\
F_{1}^{d} & =\left(D \tau^{-1} D+D^{2}-\frac{1}{2} \tau D+\frac{3}{2} D\right) \delta_{1} f .
\end{aligned}
$$

Case 2: Assume that $f$ does not depend on $D$. Then $F_{-1}^{d}=F_{0}^{d}+D \delta_{1} f=F_{0}^{d}$, and equating the diagonals in (46) we get $F_{-1}^{d}=F_{0}^{d}=\mathbf{0}$. Doing the same with (45), we have that $F_{1}^{d}=\mathbf{0}$ also.

Now, assume that $f$ depends on $D$, while $h$ doesn't. Then, both $(\nabla \mathscr{F})_{-1}$ and $\left(\nabla \mathscr{F}-\tau \nabla^{\prime} \mathscr{F}\right)_{1}$, whose only nonconstant block is given by

$$
F_{1}-\tau\left(\widehat{D}\left(F_{1}+K_{1} F_{0}+F_{0}^{T} K_{1}-K_{1} F_{-1} K_{1}\right) \hat{D}\right),
$$

are diagonal, while $(\nabla \mathscr{H})_{-1}$ and $\left(\nabla \mathscr{H}-\tau \nabla^{\prime} \mathscr{H}\right)_{1}$ are diagonal free. Therefore as one can readily see from the formula in (21),

$$
\{f, h\}_{\text {inv }}=0 .
$$

If both $f$ and $h$ depend on $D$ only, we can substitute (47)-(48)-(49) in (21) to find the double reduction. It is given by

$$
\begin{aligned}
& \{f, h\}_{\text {inv }}(D) \\
& =\frac{1}{2}\left\langle\delta_{1} h, D\left(\left(\tau^{-1}-\tau\right) D+D\left(\tau^{-1}-\tau\right)+2\left(\tau^{-1}-\tau\right)+\tau^{-1} D \tau^{-1}-\tau D \tau\right) D \delta_{1} f\right\rangle,
\end{aligned}
$$

which is a decoupled system of second Hamiltonian structures for the Volterra lattice, as stated. 
We can now see where the relation $\boldsymbol{v}_{s}=\frac{1}{2}\left(I+\tau^{-1}\right) D_{s} \delta_{s} f$ came from: $F_{-1}=$ $\frac{1}{2}(\tau+1) D \delta_{1} f$ and $\tau \boldsymbol{v}_{s}=F_{-1}$, according to (23).

\section{Conclusion and further study}

In this paper we have shown that, if $G$ is semisimple and $\mathfrak{g}$ is a $|1|$-graded Lie algebra with a parabolic gradation compatible with a second grading of the form (17), then the moduli space of polygons in $G / P$ is endowed with a natural Poisson bracket that can be linked to invariantizations of polygon evolutions. As an example we described in detail the case of polygons of Lagrangian subspaces in $\mathbb{R}^{2 n}$. We show that under some nondegeneracy conditions the Poisson bracket can be restricted further to a certain submanifold of Lagrangian planes, and that on this submanifold the eigenvalues of the Lagrangian Schwarzian difference evolve following a Hamiltonian evolution, one that becomes a decoupled system of Volterra equations when a proper Hamiltonian is chosen.

In the continuous case, the existence of a Poisson structure on the moduli space of curves is guaranteed not only for the case of $|1|$-graded Lie algebras but also for general homogeneous manifolds of the form $G / H$ with $G$ semisimple [Marí Beffa 2010] and for semidirect products [Marí Beffa 2006]. It is well possible that the same is true for the discrete counterpart, but the discrete case is more difficult to study. The main obstacle is the need to rely on $R$-matrices to define the Poisson Lie group at the beginning of our construction. If we consider a general case $G / H$, with $\hat{\mathfrak{h}}$ being the Lie algebra of $H$, then to be able to use these Poisson structures we will need to coordinate $\hat{\mathfrak{h}}$ with a gradation of the form (17), with $\hat{\mathfrak{h}}^{0} \subset \mathfrak{g}_{+}$: on the one hand (17) is used to define the $R$-matrix, and on the other hand $\hat{\mathfrak{h}}$ is used for the reduction itself, so both need to be coordinated throughout calculations. Not only that, if $m$ is a linear complement for $\hat{\mathfrak{h}}$, so $\mathfrak{g}=\hat{\mathfrak{h}} \oplus \mathfrak{m}$, in order for the proof of Theorem 3.4 to go through, one can check that we would need the condition $\mathfrak{m}^{*} \cap \mathfrak{m}=\mathbf{0}$. At first sight, this seems to not be always possible since choosing $\hat{\mathfrak{h}}=\mathfrak{g}_{1}$ (instead of $\hat{\mathfrak{h}}=\mathfrak{g}_{1} \oplus \mathfrak{g}_{0}$ ) provides a simple counterexample. Furthermore, in the general case, the action of $G$ on $G / H$ will also determine whether or not the bracket reduces. Indeed, the fact that the infinitesimal action was either constant, linear or quadratic, depending on which subgroup of $G$ we were using, was fundamental to the admissibility of $\mathfrak{p}$ (we need the action to vanish at zero, and the derivative of the infinitesimal action of $\hat{\mathfrak{h}}^{0}$ to also vanish at zero). Hence, one will have to decide which actions qualify and which ones don't. Thus, although a more general theorem is true for those other cases that satisfy these three conditions, it would not be as general as the theorem for curve evolutions. Surprisingly, the case of the homogeneous 2-sphere $\mathrm{SO}(3) / \mathrm{SO}(2)$ does not satisfy these three conditions (one can check that if $\mathfrak{m}$ is a linear complement $\mathfrak{m}^{*} \cap \mathfrak{m} \neq \mathbf{0}$ ), 
but nevertheless in [Mansfield et al. 2013], we described polygon evolutions on the 2-sphere $\mathrm{SO}(3) / \mathrm{SO}(2)$ inducing an equation of Volterra type on the discrete curvature of the polygon. Thus, perhaps a somehow different approach is needed to increase the generality. Work in that direction is under way.

A different and very interesting question is how one can get a second Hamiltonian structure, a companion for the reduction, to be used for integrability of difference evolutions. This point is not at all clear: in the continuous case it is know that it comes from a reduction of a second Hamiltonian structure (see [Marí Beffa 2010]), but it is also known that this second structure is not always reducible (a counterexample can be found in [Marí Beffa 2007]). No such natural second structure seems to exist in the discrete case and the situation becomes more murky. In [Marí Beffa and Wang 2013] we showed that in the case of $\mathbb{R P}^{n}$, even though the right bracket (the portion of the Sklyanin bracket involving right gradients only) is not Poisson, when reduced to $G^{N} / P^{N}$ the result is Poisson and a second Hamiltonian structure for integrable discretizations of $W_{n}$-algebras. It seems to be a similar situation as having the Sklyanin bracket reduce to a Poisson bracket, even though it is not a Poisson bracket with our choice of parabolic gradation. Thus, perhaps there is an underlying bracket that coordinates with the right bracket to give the same result, but what bracket this might be is unknown to us.

\section{References}

[Bobenko and Suris 2008] A. I. Bobenko and Y. B. Suris, Discrete differential geometry: integrable structure, Graduate Studies in Mathematics 98, American Mathematical Society, Providence, RI, 2008. MR 2010f:37125 Zbl 1158.53001

[Boutin 2002] M. Boutin, "Joint invariant signatures for curve recognition", pp. 37-52 in Inverse problems, image analysis, and medical imaging (New Orleans, LA, 2001), edited by M. Z. Nashed and O. Scherzer, Contemp. Math. 313, American Mathematical Society, Providence, RI, 2002. MR 2003m:53023 Zbl 1063.68085

[Calini et al. 2009] A. Calini, T. Ivey, and G. Marí Beffa, "Remarks on KdV-type flows on star-shaped curves", Phys. D 238:8 (2009), 788-797. MR 2010m:37127 Zbl 1218.37102

[Chou and $\mathrm{Qu}$ 2002] K.-S. Chou and C. Qu, "Integrable equations arising from motions of plane curves”, Phys. D 162:1-2 (2002), 9-33. MR 2003c:37106 Zbl 0987.35139

[Chou and Qu 2003] K.-S. Chou and C. Qu, "Integrable equations arising from motions of plane curves, II”, J. Nonlinear Sci. 13:5 (2003), 487-517. MR 2005d:37155 Zbl 1045.35063

[Drinfeld 1983] V. G. Drinfeld, "Hamiltonian structures on Lie groups, Lie bialgebras and the geometric meaning of classical Yang-Baxter equations", Dokl. Akad. Nauk SSSR 268:2 (1983), 285287. In Russian; translated in Sov. Math. Dokl. 27 (1983), 68-71. MR 84i:58044 Zbl 0526.58017

[Frenkel et al. 1998] E. Frenkel, N. Reshetikhin, and M. A. Semenov-Tian-Shansky, "DrinfeldSokolov reduction for difference operators and deformations of $\mathcal{W}$-algebras, I: The case of Virasoro algebra”, Comm. Math. Phys. 192:3 (1998), 605-629. MR 2000b:17031 Zbl 0916.17020

[Khanizadeh et al. 2013] F. Khanizadeh, A. V. Mikhailov, and J. P. Wang, "Darboux transformations and recursion operators for differential-difference equations", Theor. Math. Phys. 177:3 (2013), 1606-1654. arXiv 1305.0588 
[Khesin and Soloviev 2013] B. Khesin and F. Soloviev, "Integrability of higher pentagram maps", Math. Ann. 357:3 (2013), 1005-1047. MR 3118623 Zbl 1280.37056

[Kobayashi and Nagano 1964] S. Kobayashi and T. Nagano, "On filtered Lie algebras and geometric structures, I”, J. Math. Mech. 13:5 (1964), 875-907. MR 29 \#5961 Zbl 0142.19504

[Kobayashi and Nagano 1965] S. Kobayashi and T. Nagano, "On filtered Lie algebras and geometric structures, II”, J. Math. Mech. 14:3 (1965), 513-521. MR 32 \#2512 Zbl 0163.28103

[Mansfield et al. 2013] E. Mansfield, G. Marí Beffa, and J. P. Wang, "Discrete moving frames and discrete integrable systems", Found. Comput. Math. 13:4 (2013), 545-582. MR 3085678 Zbl 1279.14045

[Marí Beffa 1999] G. Marí Beffa, "The theory of differential invariants and KdV Hamiltonian evolutions”, Bull. Soc. Math. France 127:3 (1999), 363-391. MR 2001m:37142 Zbl 1053.37046

[Marí Beffa 2006] G. Marí Beffa, "Poisson geometry of differential invariants of curves in some nonsemisimple homogeneous spaces", Proc. Amer. Math. Soc. 134:3 (2006), 779-791. MR 2006f:53122 Zbl 1083.37053

[Marí Beffa 2007] G. Marí Beffa, "On completely integrable geometric evolutions of curves of Lagrangian planes”, Proc. Roy. Soc. Edinburgh Sect. A 137:1 (2007), 111-131. MR 2008i:37144 Zbl 1130.37032

[Marí Beffa 2010] G. Marí Beffa, "Bi-Hamiltonian flows and their realizations as curves in real semisimple homogeneous manifolds", Pacific J. Math. 247:1 (2010), 163-188. MR 2012m:37118 Zbl 1213.37096

[Marí Beffa 2013] G. Marí Beffa, "On generalizations of the pentagram map: discretizations of AGD flows”, J. Nonlinear Sci. 23:2 (2013), 303-334. MR 3041627 Zbl 06175210

[Marí Beffa and Wang 2013] G. Marí Beffa and J. P. Wang, "Hamiltonian evolutions of twisted polygons in $\mathbb{R P}^{n}$ ", Nonlinearity 26:9 (2013), 2515-2551. MR 3093293 Zbl 06214273

[Marshall 2010] I. Marshall, "Poisson reduction of the space of polygons", preprint, 2010. arXiv $1007.1952 \mathrm{v} 1$

[Ochiai 1970] T. Ochiai, "Geometry associated with semisimple flat homogeneous spaces", Trans. Amer. Math. Soc. 152 (1970), 159-193. MR 44 \#2160 Zbl 0205.26004

[Ovsienko 1993] V. Ovsienko, "Lagrange Schwarzian derivative and symplectic Sturm theory", Ann. Fac. Sci. Toulouse Math. (6) 2:1 (1993), 73-96. MR 94g:58079 Zbl 0780.34004

[Ovsienko et al. 2010] V. Ovsienko, R. E. Schwartz, and S. Tabachnikov, "The pentagram map: a discrete integrable system”, Comm. Math. Phys. 299:2 (2010), 409-446. MR 2012a:37140 Zbl 1209.37063

[Ovsienko et al. 2013] V. Ovsienko, R. E. Schwartz, and S. Tabachnikov, "Liouville-Arnold integrability of the pentagram map on closed polygons", Duke Math. J. 162:12 (2013), 2149-2196. MR 3102478 Zbl 06218376

[Semenov-Tian-Shansky 1985] M. A. Semenov-Tian-Shansky, "Dressing transformations and Poisson group actions”, Publ. Res. Inst. Math. Sci. 21:6 (1985), 1237-1260. MR 88b:58057 Zbl 0673.58019

[Soloviev 2013] F. Soloviev, "Integrability of the pentagram map", Duke Math. J. 162:15 (2013), 2815-2853. MR 3161305 Zbl 1282.14061

[Terng and Thorbergsson 2001] C.-L. Terng and G. Thorbergsson, "Completely integrable curve flows on adjoint orbits", Results Math. 40:1-4 (2001), 286-309. MR 2002k:37141 Zbl 1023.37041

[Terng and Uhlenbeck 2006] C.-L. Terng and K. Uhlenbeck, "Schrödinger flows on Grassmannians", pp. 235-256 in Integrable systems, geometry, and topology, edited by C.-L. Terng, AMS/IP Stud. Adv. Math. 36, American Mathematical Society, Providence, RI, 2006. MR 2007a:37079 Zbl 1110.37056 
[Wang 2002] J. P. Wang, "Generalized Hasimoto transformation and vector sine-Gordon equation", pp. 276-283 in Symmetry and perturbation theory (Cala Gonone, 2002), edited by S. Abenda et al., World Scientific, River Edge, NJ, 2002. MR 2004c:37172

Received May 20, 2013. Revised April 10, 2014.

Gloria MARÍ BeFFA

MATHEMATICS DEPARTMENT

UNIVERSITY OF WISCONSIN

480 LINCOLN DRIVE

MADISON, WI 53706

UNITED STATES

maribeff@math.wisc.edu 


\title{
PACIFIC JOURNAL OF MATHEMATICS
}

\author{
msp.org/pjm
}

Founded in 1951 by E. F. Beckenbach (1906-1982) and F. Wolf (1904-1989)

\section{EDITORS}

Don Blasius (Managing Editor)

Department of Mathematics

University of California

Los Angeles, CA 90095-1555

blasius@math.ucla.edu

\author{
Paul Balmer \\ Department of Mathematics \\ University of California \\ Los Angeles, CA 90095-1555 \\ balmer@math.ucla.edu \\ Robert Finn \\ Department of Mathematics \\ Stanford University \\ Stanford, CA 94305-2125 \\ finn@math.stanford.edu \\ Sorin Popa \\ Department of Mathematics \\ University of California \\ Los Angeles, CA 90095-1555 \\ popa@math.ucla.edu
}

\author{
Vyjayanthi Chari \\ Department of Mathematics \\ University of California \\ Riverside, CA 92521-0135 \\ chari@math.ucr.edu \\ Kefeng Liu \\ Department of Mathematics \\ University of California \\ Los Angeles, CA 90095-1555 \\ liu@math.ucla.edu \\ Jie Qing \\ Department of Mathematics \\ University of California \\ Santa Cruz, CA 95064 \\ qing@ cats.ucsc.edu
}

\section{PRODUCTION}

Silvio Levy, Scientific Editor, production@msp.org

\section{SUPPORTING INSTITUTIONS}

ACADEMIA SINICA, TAIPEI

CALIFORNIA INST. OF TECHNOLOGY

INST. DE MATEMÁTICA PURA E APLICADA

KEIO UNIVERSITY

MATH. SCIENCES RESEARCH INSTITUTE

NEW MEXICO STATE UNIV.

OREGON STATE UNIV.

\author{
STANFORD UNIVERSITY \\ UNIV. OF BRITISH COLUMBIA \\ UNIV. OF CALIFORNIA, BERKELEY \\ UNIV. OF CALIFORNIA, DAVIS \\ UNIV. OF CALIFORNIA, LOS ANGELES \\ UNIV. OF CALIFORNIA, RIVERSIDE \\ UNIV. OF CALIFORNIA, SAN DIEGO \\ UNIV. OF CALIF., SANTA BARBARA
}

\author{
Daryl Cooper \\ Department of Mathematics \\ University of California \\ Santa Barbara, CA 93106-3080 \\ cooper@math.ucsb.edu \\ Jiang-Hua Lu \\ Department of Mathematics \\ The University of Hong Kong \\ Pokfulam Rd., Hong Kong \\ jhlu@maths.hku.hk \\ Paul Yang \\ Department of Mathematics \\ Princeton University \\ Princeton NJ 08544-1000 \\ yang@math.princeton.edu
}

These supporting institutions contribute to the cost of publication of this Journal, but they are not owners or publishers and have no responsibility for its contents or policies.

See inside back cover or msp.org/pjm for submission instructions.

The subscription price for 2014 is US $\$ 410 /$ year for the electronic version, and \$535/year for print and electronic.

Subscriptions, requests for back issues and changes of subscribers address should be sent to Pacific Journal of Mathematics, P.O. Box 4163, Berkeley, CA 94704-0163, U.S.A. The Pacific Journal of Mathematics is indexed by Mathematical Reviews, Zentralblatt MATH, PASCAL CNRS Index, Referativnyi Zhurnal, Current Mathematical Publications and Web of Knowledge (Science Citation Index).

The Pacific Journal of Mathematics (ISSN 0030-8730) at the University of California, c/o Department of Mathematics, 798 Evans Hall \#3840, Berkeley, CA 94720-3840, is published twelve times a year. Periodical rate postage paid at Berkeley, CA 94704, and additional mailing offices. POSTMASTER: send address changes to Pacific Journal of Mathematics, P.O. Box 4163, Berkeley, CA 94704-0163.

PJM peer review and production are managed by EditFLOW ${ }^{\circledR}$ from Mathematical Sciences Publishers.

\section{PUBLISHED BY}

\section{mathematical sciences publishers \\ nonprofit scientific publishing}

http://msp.org/

(C) 2014 Mathematical Sciences Publishers 


\section{PACIFIC JOURNAL OF MATHEMATICS}

Volume $270 \quad$ No. $2 \quad$ August 2014

Disjointification inequalities in symmetric quasi-Banach spaces and 257 their applications

SERgey Astashinin, Fedor A. SukocheV and DMitriy

ZANIN

Hamiltonian evolutions of twisted polygons in parabolic manifolds:

287

The Lagrangian Grassmannian

GLORIA MARÍ BEFFA

On Schwarz-Christoffel mappings

MARTIN CHUAQUi and CHRISTIAN POMMERENKE

Vanishing viscosity in the plane for nondecaying velocity and vorticity, II

ELAINE COZZI

Affine quantum Schur algebras and affine Hecke algebras

QIANG FU

On the classification of Killing submersions and their isometries

JosÉ M. MANZANO

Locally Lipschitz contractibility of Alexandrov spaces and its applications

Ayato Mitsuishi and TAKaO Yamaguchi

Sequences of open Riemannian manifolds with boundary

RAQUEL PERALES and CHRISTINA SORMANI

Invariant differential operators on a class of multiplicity-free spaces 